\title{
Metabotypes with elevated protein and lipid catabolism and inflammation precede clinical mastitis in prepartal transition dairy cows
}

\author{
F. Zandkarimi, ${ }^{*}$ J. Vanegas, $†$ X. Fern, $\ddagger$ C. S. Maier, ${ }^{\star} \S$ and G. Bobe§\# ${ }^{1}$ \\ *Department of Chemistry, \\ †Department of Veterinary Clinical Sciences, \\ †Department of Electrical Engineering and Computer Science, \\ §Linus Pauling Institute, and \\ \#Department of Animal and Rangeland Sciences, Oregon State University, Corvallis 97331
}

\begin{abstract}
Clinical mastitis (CM), the most prevalent and costly disease in dairy cows, is diagnosed most commonly shortly after calving. Current indicators do not satisfactorily predict CM. This study aimed to develop a robust and comprehensive mass spectrometry-based metabolomic and lipidomic workflow using untargeted ultra-performance liquid chromatography high-resolution mass spectrometry for predictive biomarker detection. Using a nested case-control design, we measured weekly during the prepartal transition period differences in serum metabolites, lipids, inflammation markers, and minerals between clinically healthy Holstein dairy cows diagnosed with mastitis postcalving $(\mathrm{CMP} ; \mathrm{n}=8$; CM diagnosis $\mathrm{d} 1=3$ cows, $\mathrm{d} 2=2$ cows, $\mathrm{d} 4=1$ cow; d $25=1$ cow, and d $43=1$ cow that had subclinical mastitis since d 3 ) or not (control; $\mathrm{n}=9$ ). The largest fold differences between CMP and control cows during the prepartal transition period were observed for $3^{\prime}$-sialyllactose in serum. Seven metabolites $(N$-methylethanolamine phosphate, choline, phosphorylcholine, free carnitine, trimethyl lysine, tyrosine, and proline) and 3 metabolite groups (carnitines, AA metabolites, and water-soluble phospholipid metabolites) could correctly classify cows for their future CM status at both 21 and $14 \mathrm{~d}$ before calving. Biochemical analysis using lipid and metabolite-specific commercial diagnostic kits supported our mass spectrometry-based omics results and additionally showed elevated inflammatory markers (serum amyloid A and visfatin) in CMP cows. In conclusion, metabolic phenotypes (i.e., metabotype) with elevated protein and lipid metabolism and inflammation may precede $\mathrm{CM}$ in prepartal transition dairy cows. The discovered serum metabolites and lipids may assist in predictive diagnostics, prevention strategies,
\end{abstract}

Received October 11, 2017.

Accepted January 15, 2018.

${ }^{1}$ Corresponding author: gerd.bobe@oregonstate.edu and early treatment intervention against $\mathrm{CM}$, and thereby improve cow health and welfare.

Key words: clinical mastitis, lipidomic, metabolomic, transition period, 3'-sialyllactose

\section{INTRODUCTION}

Bovine mastitis is an inflammatory response of the mammary gland, which is primarily caused by bacterial infections (Eberhardt, 1996; Viguier et al., 2009). Bovine mastitis can be subdivided into clinical (CM) and subclinical mastitis (SCM); CM can be diagnosed by visible changes in milk consistency and mammary gland appearance (redness, swelling, heat, or pain) or both, and SCM can be diagnosed by elevated SCC in milk (>200,000 cells/mL milk) in the absence of visible changes in milk and udder appearance (Eberhardt, 1996; Sharma et al., 2011). Bovine CM is one of the most prevalent and costly clinical diseases in dairy cows, which makes it economically important to the dairy industry. In the United States, about $16.5 \%$ of the dairy cows have $\mathrm{CM}$ in the first $30 \mathrm{~d}$ of lactation (USDA, 2009) and the incurred cost is about $\$ 440$ per case (Kelton et al., 1998; Rollin et al., 2015); therefore, prevention and early treatment of CM are a priority.

Traditional CM indicator studies focus on indicators in milk at the onset of CM, including SCC, serum proteins, enzymes, electrolytes, degradation products of milk proteins, and acute phase proteins (Lai et al., 2009; Sundekilde et al., 2013). In recent years, this research has been extended to metabolomics approaches to discover indicators in infected bovine milk, which can aid in the detection, differential diagnosis of $\mathrm{CM}$ based on pathogen, and to examine the pathophysiology of CM (Mansor et al., 2013; Thomas et al., 2016; $\mathrm{Xi}$ et al., 2017). Dairy cows are most susceptible to naturally occurring CM within the first weeks after calving; however, the infection may occur during the close-up period or around calving (Rollin et al., 2015), when milk samples are not available (Hurley and Theil, 
2011). Multiple studies have become available during recent years that used blood samples collected during the last 2 mo before and after calving for the discovery of predictive biomarker of various diseases in early-lactation dairy cows (Imhasly et al., 2015; Dervishi et al., 2017; Zhang et al., 2017); however, to the best of our knowledge, none looked at predictive serum indicators of CM. Dervishi et al. $(2015,2017)$ reported that cows subsequently developing SCM had elevated serum concentrations of inflammatory markers, monosaccharides, and $\mathrm{AA}$ and their metabolites 4 wk before calving.

Our hypothesis was that untargeted ultra-performance liquid chromatography high resolution mass spectrometry (UPLC-MS ${ }^{\mathrm{E}}$ ) can be used to discover serum metabolites and lipids that precede $\mathrm{CM}$ during the close-up period in dairy cows. To accomplish our goal, our major objectives were (1) to develop a robust and comprehensive, untargeted MS-based metabolomic and lipidomic workflow for biomarker detection in serum, which could be also applied to other biological fluids; (2) to identify serum metabolites, lipids, minerals, and inflammatory markers, individually or as group, that can classify during the close-up period dairy cows that develop CM in early lactation; and (3) to describe temporal changes in serum metabolites, lipids, minerals, and inflammatory markers during the prepartal transition period.

\section{MATERIALS AND METHODS}

This study was part of a prospective study designed to identify predictive serum indicators of periparturient diseases in dairy cows. All procedures involving animals were approved by the Oregon State University Institutional Animal Care and Use Committee (ACUP Number 3991).

\section{Study Design and Animal Management}

The study cohort consisted of 161 purebred Holstein cows from a 1,000-head commercial dairy farm in Oregon's Central Willamette Valley; cows had 1 to 6 completed lactations and were free of clinical diseases, including abnormal mammary gland appearance (tender, painful or warm to touch, swelling, hardness, or skin redness), 4 wk before expected calving date. Using a nested case-control design, we identified 8 cows that developed CM postcalving (CMP) and matched them by parity (mean $\pm \mathrm{SD}$, range; $\mathrm{CMP}=1.88 \pm 0.64,1-3$ completed lactations; control $=1.44 \pm 0.73,1-3$ completed lactations), BCS (control $=3.68 \pm 0.19,3.3-3.9$; $\mathrm{CMP}=3.75 \pm 0.29,3.3-4.1)$, and calving season with 9 cows that remained free of clinical diseases (control) as well as subclinical ketosis and hypocalcemia during the first $49 \mathrm{~d}$ after calving. Clinical mastitis was diagnosed based on daily test for abnormal milk consistency (e.g., flakes, clots) and mammary gland appearance (redness, swelling, heat, or pain). If abnormal milk consistency, mammary gland appearance, or both were detected, a milk sample was collected and on-farm culturing with blood agar was performed. All CMP cows except 2 were diagnosed with $\mathrm{CM}$ within $4 \mathrm{~d}$ after calving: $\mathrm{d} 1=3$ cows, d $2=2$ cows, and d $4=1$ cow. Another CMP cow had SCM ( SCC >1,000,000 cells $/ \mathrm{mL}$ ) directly after calving and was diagnosed with CM $43 \mathrm{~d}$ after calving. The only CMP cow without elevated SCC at the beginning of lactation was diagnosed with $\mathrm{CM}$ at $25 \mathrm{~d}$ after calving. To identify serum indicators that could precede CM from various naturally occurring pathogens rather than indicators specific to 1 type of pathogens, we selected cows that differed in pathogens based on cultured growth (gram-positive $=3$ cows; gram-negative $=3$ cows; no cultured growth $=2$ cows). Within $1 \mathrm{~d}$ of CM diagnosis, cows were treated based on their cultured growth. Three CMP cows (6, 9, and 10 mo prior) and 1 control cow (11 mo prior) had an episode of $\mathrm{CM}$ in the previous 12 mo. Based on the cultured growth, the current CM was from a different pathogen than the prior CM. To avoid confounding with other diseases, cows that developed other concurrent clinical diseases besides CM were excluded from this nested case-control study.

Management of the cows and animal health surveillance and disease treatment has been described in detail previously (Qu et al., 2014); additional information is provided in Supplemental File S1 (https://doi .org/10.3168/jds.2017-13977). Starting 28 d before the expected calving date, BCS of cows were scored once weekly by 3 trained independent evaluators until $4 \mathrm{wk}$ after calving (Edmonson et al., 1989). Before calving, cows were housed in a straw-bedded freestall barn and were fed once in the morning $(0730 \mathrm{~h})$, a TMR based on corn, corn silage, and alfalfa and triticale hay that met NRC guidelines (NRC, 2001), as summarized in Supplemental Table S1 (https://doi.org/10.3168/ jds.2017-13977). After calving, cows were housed in freestall pens with slatted floors and were fed in head gates around 0800 and $1330 \mathrm{~h}$ a TMR based on corn, corn silage, and alfalfa hay (Supplemental Table S1) that met NRC guidelines (NRC, 2001).

\section{Sample Collection}

Blood samples were taken from the coccygeal vein or artery at 3 ( -24 to $-18 \mathrm{~d}), 2(-17$ to $-11 \mathrm{~d})$, and 1 wk $(-10$ to $-4 \mathrm{~d})$ before calving and the morning after calving within $10 \mathrm{~min}$ after morning feeding. We chose serum rather than plasma as biological matrix 
because of higher metabolite signal intensities with serum than plasma using LC-MS (Yu et al., 2011; Lin et al., 2014); serum is the preferred biological matrix in recent metabolomic studies in dairy cows (Huber et al., 2016; Dervishi et al., 2017; Zhang et al., 2017). Serum samples were prepared by centrifugation at $1,600 \times g$ for $20 \mathrm{~min}$ at $4^{\circ} \mathrm{C}$ and stored at $-80^{\circ} \mathrm{C}$ until biochemical, metabolomic, and lipidomic analyses.

\section{Sample Preparation for Mass Spectrometric Analysis}

For metabolomic analysis, metabolites were extracted from serum samples by 1:4 dilution with ethanol/methanol $(1: 1, \mathrm{vol} / \mathrm{vol}$ ), as described previously (Kirkwood et al., 2013). Briefly, each sample was vortexed and centrifuged at $16,000 \times g$ for $20 \mathrm{~min}$ at $4^{\circ} \mathrm{C}$ to remove precipitated proteins. Supernatant was transferred to a glass vial for MS analysis.

For lipidomic analysis, 3 sample preparation methods were initially compared, as described in detail in Supplemental File S2 (https://doi.org/10.3168/jds.2017 -13977). The isopropyl alcohol-induced (IPA) protein precipitation method was used for all subsequent lipidomic analyses. Lipids were extracted from serum samples by $1: 3$ dilutions with $240 \mu \mathrm{L}$ of chilled IPA $(1: 3 \mathrm{vol} / \mathrm{vol})$ containing nonendogenous lipid standards with final concentrations of $1 \mathrm{mg} / \mathrm{L}$. Each sample was vortex-mixed vigorously for $5 \mathrm{~min}$ and incubated on ice for $10 \mathrm{~min}$. Samples were stored at $-20^{\circ} \mathrm{C}$ overnight to enhance protein precipitation. On the following day, each sample was vortex-mixed for $5 \mathrm{~min}$ and centrifuged at $16,000 \times g$ for $15 \mathrm{~min}$ at $4^{\circ} \mathrm{C}$. Supernatant was transferred to a fresh tube and stored at $-80^{\circ} \mathrm{C}$ until MS analysis.

\section{Metabolomic Analysis}

Metabolomic analysis was performed by injecting 3 $\mu \mathrm{L}$ of each sample using the flow-through needle mode on Waters Acquity UPLC I class system (Waters, Milford, MA) coupled to a Synapt G2 HDMS mass spectrometer (Waters). Metabolites were separated on an Acquity UPLC BEH amide column $(2.1 \mathrm{~mm} \times$ $150 \mathrm{~mm}, 1.7 \mu \mathrm{m}$, Waters). Mobile phase A consisted of $\mathrm{H}_{2} \mathrm{O} /$ acetonitrile (95:5, vol/vol), and mobile phase B was $\mathrm{H}_{2} \mathrm{O} /$ acetonitrile $(5: 95$, vol/vol); both mobile phases contained $0.1 \%$ formic acid. The elution gradient was $0 \mathrm{~min}, 99 \% \mathrm{~B} ; 7.5 \mathrm{~min}, 40 \% \mathrm{~B} ; 9 \mathrm{~min}, 99 \% \mathrm{~B} ; 10 \mathrm{~min}$, $99 \% \mathrm{~B}$; and $12 \mathrm{~min}, 99 \% \mathrm{~B}$. The flow rate was $0.4 \mathrm{~mL} /$ min. The temperature of the column compartment was set to $45^{\circ} \mathrm{C}$. The autosampler tray was maintained at $6^{\circ} \mathrm{C}$. Sample analysis was performed over a 12 -min total run time.
The Synapt G2 mass spectrometer was operated in the $\mathrm{MS}^{\mathrm{E}}$ mode. All analyses were conducted in both positive and negative electrospray ionization modes. Mass spectral data were acquired from $m / z 50$ to 1200. A capillary voltage of $( \pm) 2.5 \mathrm{kV}$ and a sampling cone voltage of $( \pm) 35 \mathrm{~V}$ were used. Source and desolvation temperature were kept at 100 and $400^{\circ} \mathrm{C}$, respectively. Nitrogen was used as desolvation gas with a flow rate of $650 \mathrm{~L} / \mathrm{hr}$ in the positive ionization mode, and $750 \mathrm{~L} /$ $\mathrm{hr}$ in the negative ionization mode. Dependent on the ionization mode the protonated molecular ion of leucine enkephalin, $[\mathrm{M}+\mathrm{H}]^{+}(m / z 556.2771)$ or the deprotonated molecular ion $[\mathrm{M}-\mathrm{H}]^{-}(\mathrm{m} / z$ 554.2615) was used as a lock mass for accurate mass measurement. Leucine enkephalin, dissolved in 50\% aqueous acetonitrile containing $0.1 \%$ formic acid at a concentration of $2 \mathrm{ng} /$ $\mu \mathrm{L}$, was introduced with a flow rate of $5 \mu \mathrm{L} / \mathrm{min}$. The lock mass was acquired for $0.3 \mathrm{~s}$ and repeated every 10 $\mathrm{s}$ in a separate acquisition channel. In $\mathrm{MS}^{\mathrm{E}}$ mode, the low-energy function was set to $4 \mathrm{eV}$ in the transfer cell (first function), and for collision-induced dissociation the energy in the transfer cell (second function) was increased from 15 to $35 \mathrm{eV}$.

\section{Lipidomic Analysis}

Lipidomic analysis was performed by injecting $5 \mu \mathrm{L}$ of each sample using the flow-through needle mode on Waters Acquity UPLC I class system coupled to a Synapt G2 HDMS mass spectrometer (Waters). Lipids were separated on an Acquity HSS T3 column (2.1 mm $\times 100 \mathrm{~mm}, 1.8 \mu \mathrm{m}$, Waters). Mobile phase A consisted of acetonitrile $/ \mathrm{H}_{2} \mathrm{O}(40: 60, \mathrm{vol} / \mathrm{vol})$, and mobile phase $B$ was IPA/acetonitrile $/ \mathrm{H}_{2} \mathrm{O}$ (85:10:5, $\left.\mathrm{vol} / \mathrm{vol} / \mathrm{vol}\right)$; both mobile phases contained $10 \mathrm{~m} M$ ammonium acetate and $0.1 \%$ acetic acid. The elution gradient was 0 $\min , 40 \% \mathrm{~B} ; 1 \mathrm{~min}, 40 \% \mathrm{~B} ; 11 \mathrm{~min}, 100 \% \mathrm{~B} ; 14 \mathrm{~min}$, $100 \% \mathrm{~B} ; 15 \mathrm{~min}, 40 \% \mathrm{~B}$; and $16 \mathrm{~min}, 40 \% \mathrm{~B}$ (1 min for re-equilibration time). The flow rate was $0.4 \mathrm{~mL} / \mathrm{min}$. The temperature of the column compartment was set to $55^{\circ} \mathrm{C}$. The autosampler tray was maintained at $6^{\circ} \mathrm{C}$. Sample analysis was performed over a 15-min total run time. The Synapt G2 mass spectrometer was operated in the data-independent $\left(\mathrm{MS}^{\mathrm{E}}\right)$ mode using the same settings as described above for the metabolomics analysis. The exception is that for collision-induced dissociation the energy in the transfer cell (second function) was increased from 25 to $60 \mathrm{eV}$.

Detailed information about quality control (QC), metabolite and lipid validation are provided in Supplemental File S3 (https://doi.org/10.3168/jds.2017 -13977). Detailed information about data processing and feature annotation for lipidomic and metabolomics 
analysis is provided in Supplemental File S4 (https:// doi.org/10.3168/jds.2017-13977).

\section{Biochemical Analysis}

Serum concentrations of total glucose, BHB, fatty acids, urea nitrogen, haptoglobin, visfatin, cholesterol, $\alpha$-tocopherol, calcium, magnesium, and phosphorus were determined as reported previously $(\mathrm{Qu}$ et al., 2013, 2014; Fadden and Bobe, 2015). Serum concentrations of serum amyloid A (SAA) were determined using a multispecies ELISA kit (Catalog No. KAA0021; Life Technologies, Grand Island, NY), as SAA is highly conserved across species. Manufacturer's instructions (https://www.thermofisher.com/order/catalog/ product/KAA0021) were followed for chemical analysis. Absorbance was measured at $450 \mathrm{~nm}$ with a FLUOstar Omega microplate auto-reader (BMG Labtech Inc., San Francisco, CA). Serum concentration of serum tumor necrosis factor- $\alpha$ was measured with a bovine ELISA kit (Catalog No. RAB0522-1KT; Sigma Aldrich, St. Louis, MO) according to the manufacturer's instructions (https://www.sigmaaldrich.com/technical -documents/protocols/biology/elisa-protocols.html). Inter- and intra-assay coefficients of variation are in Supplemental Table S2 (https://doi.org/10.3168/jds .2017-13977).

\section{Data Processing, Statistical Analysis, and Self-Organizing Maps}

Data acquisition was performed in MassLynx (version 4.1, Waters Corp., Milford, MA) software (version 4.1) in centroid mode. Mass spectrometery data preprocessing and feature extraction were performed using an open-source XCMS package (version 1.39.4) in $\mathrm{R}$ (version 3.1.2; R Core Team, 2013) environment for peak picking, retention time alignment, and filtering, as provided under Supplemental File S4 (https:// doi.org/10.3168/jds.2017-13977). The area counts for each feature (i.e., signal intensities) in each sample were used for creating bar plots. We performed statistical analyses using MetaboAnalyst, version 2.0 (Xia et al., 2012), GraphPad Prism version 7.00 for Windows (GraphPad Software, La Jolla, CA), and SAS version 9.4 (SAS Institute Inc., Cary, NC). For metabolomic data, we normalized area counts for each metabolite in each sample using the Loess algorithm to correct for variations originating from interday running differences of the instrument platform (Ejigu et al., 2013). To compare data obtained by biochemical analysis with data obtained by metabolomics or lipidomics, Pearson correlation coefficient was used.

The potential predictive strength were evaluated by determining how accurately individual serum me- tabolites and lipids or summed biological families could classify cows for their future CM status. An area under the receiver operating curve (AUC-ROC) value of 1 indicates that an individual serum metabolite and lipid or summed biological groups could discriminate between cow groups with $100 \%$ accuracy (i.e., complete separation between groups). We also computed AUCROC for individual serum metabolites and lipids or summed biological groups that misclassified only 1 or 2 cows. We did not compute AUC-ROC for combinations of serum metabolites and lipids, as individual serum metabolites and lipids or summed biological families were sufficient to classify cows.

Significance of differences between CMP and control cows were evaluated by the nonparametric Wilcoxon rank-sum test. To correct for multiple testing, the Benjamin-Hochberg method was used to compute $q$-values (Broadhurst and Kell, 2007). The results of parametric $t$-test with Welch-Satterthwaite approximation are not shown because their variance estimates are more susceptible to outliers and violation of normality.

To evaluate differences between CMP and control cows for the whole data sets (lipidomic, metabolomic, and biochemical data), linear group differences of natural log-transformed, autoscaled data were calculated and visualized using principal component analysis (unsupervised analysis) and partial least squares discriminant analysis (PLS-DA; supervised analysis). Model validation was carried out using a cross-validation test. The goodness of fit $\left(\mathrm{R}^{2}\right)$ and predictive power of PLSDA score plots were calculated.

To evaluate temporal changes within and across CMP and control cows, a repeated-measures-in-time analysis of natural log-transformed data was conducted in PROC MIXED of SAS. Repeated measures within cows were modeled using a first-order heterogeneous variance-covariance matrix and the Kenward-Roger approximation was used to adjust the $P$-values for repeated measures of the same animal. Fixed effects were time (21, 14, $7 \mathrm{~d}$ before and directly after calving), group (control and CMP cows), and their interaction.

To visualize for the whole data sets temporal changes within and across CMP and control cows, the self-organizing maps (SOM) algorithm implemented in Gene Expression Dynamics Inspector (GEDI) software (version 2.1, https://apps.childrenshospital.org/clinical/ research/ingber/GEDI/gedihome.htm; Children's Hospital, Boston, MA) was used. For each sampling time, signal intensities of individual lipids from those lipid subclasses were exported into GEDI. Ninety (9 $\times 10)$ grid coordinates were then defined and trained by using 80 first-phase and $160 \mathrm{~s}$-phase iterations. The resulting heat maps indicate the location of clustered lipids or metabolites as series of coherent mosaic tiles. 


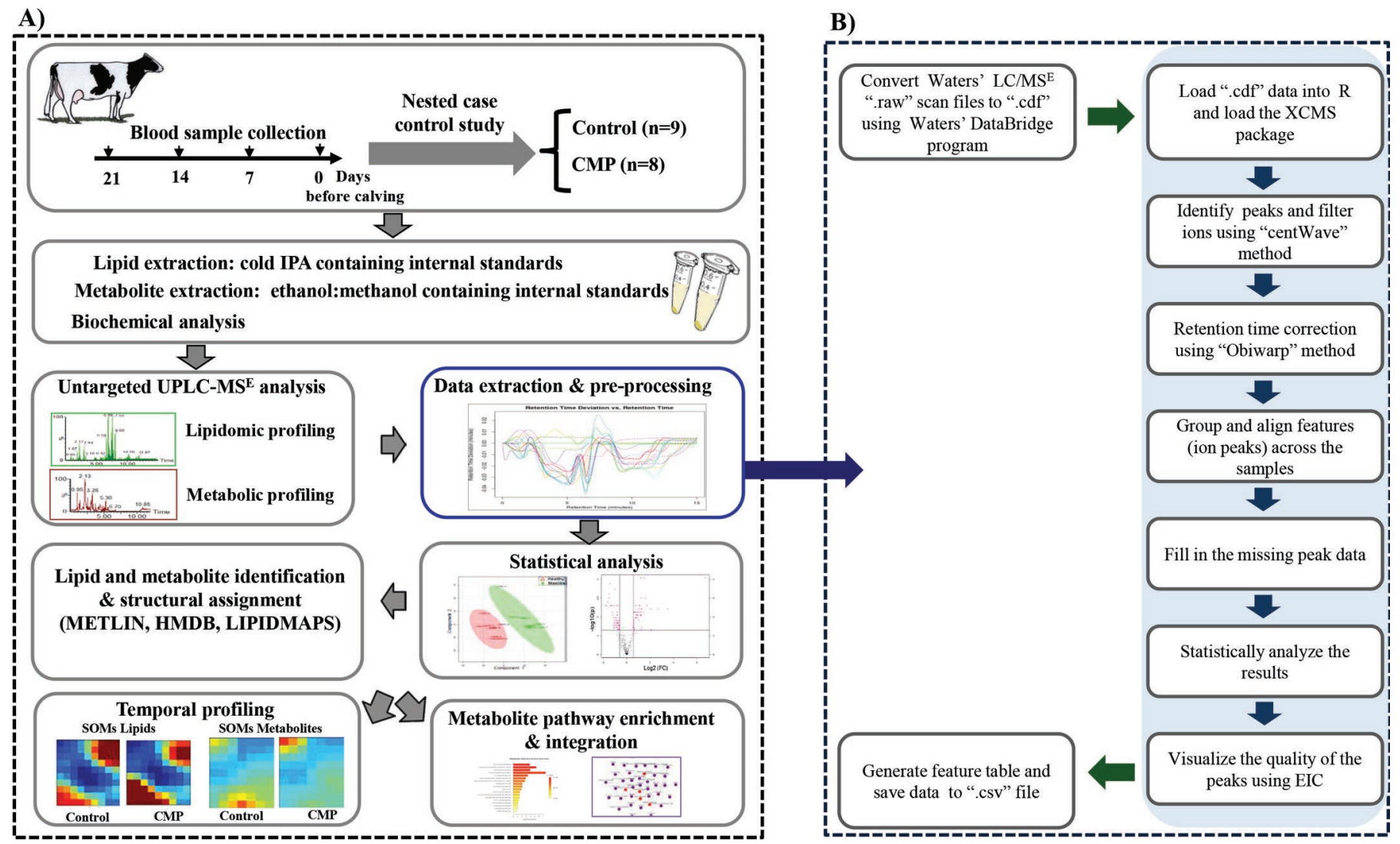

Figure 1. (A) Experimental workflow of untargeted metabolomic, lipidomic, and biochemical analyses for predictive biomarker discovery of bovine clinical mastitis during the close-up period (21, 14, 7, and $0 \mathrm{~d}$ before calving), and (B) data preprocessing pipeline using the XCMS package in R (http://www.R-project.org; EIC: extracted ion chromatogram; more details provided in Supplemental Table S5, https://doi. org/10.3168/jds.2017-13977). CMP = cows diagnosed with mastitis postcalving; IPA = isopropyl alcohol-induced; METLIN = The Metabolite and Tandem Mass Spectrometry Database; HMDB = The Human Metabolome Database; LIPIDMAPS = The Lipid Maps Database; UPLC$\mathrm{MS}^{\mathrm{E}}=$ ultra-performance liquid chromatography high resolution mass spectrometry; SOM = self-organizing maps. Color version available online.

Because of the small number of measured biochemical parameters $(n=11)$, changes could not be visualized for biochemical data.

\section{RESULTS}

\section{Analytical Workflow}

The UPLC-MS ${ }^{\mathrm{E}}$ metabolomic and lipidomic analytical workflow for predictive biomarker discovery of bovine CM is summarized in Figure 1A. Using a nested case-control design, we measured serum metabolites, lipids, inflammatory markers, and minerals at -21 , $-14,-7$, and $0 \mathrm{~d}$ from cows that in early lactation did or did not develop CM. The extracted serum metabolites and lipids were separated using hydrophilic interaction liquid chromatography and reversed-phase UPLC, respectively, and detected and quantified by high-resolution $\mathrm{MS}^{\mathrm{E}}$ and ion mobility spectrometrymass spectrometry. We used the XCMS-package in R for data extraction and preprocessing as summarized in
Figure 1B. We applied parametric and nonparametric approaches to classify cows for future CM and determine dynamic changes within and across groups, which were visualized using linear and nonlinear clustering methods. We used online databases (https://metlin .scripps.edu, http://www.lipidmaps.org, http://www .hmdb.ca) for tentative identification of features. Mass spectrometry-based omics was complemented by biochemical assays for clinical diagnostics and validation of MS-derived findings.

Within the analytical workflow, we tested 3 lipid extraction or precipitation methods: 2 lipid-liquid extraction methods, methanol/methyl tert-butyl ether and dichloromethane/methanol, and 1 protein precipitation method with IPA (Supplemental File S2; https://doi .org/10.3168/jds.2017-13977). We were able to extract the major lipid classes and subclasses with all 3 methods (Supplemental Figure S1; https://doi.org/10.3168/jds .2017-13977); the total numbers of extracted features for each of the tested protocol were around 2,250 and 1,450 ions in the positive and negative modes, respec- 
tively. The 3 extraction methods, however, resulted in differences in signal intensities for fatty acids (negative mode), triacylglycerol (TAG), and cholesteryl esters (ChoE; both in positive mode), with the IPA precipitation yielding the highest ion intensities for fatty acids, TAG, and ChoE (Supplemental Figure S1). The IPA method was used for all subsequent lipidomic analyses and provided satisfactory recovery and reproducibility coefficients of variation (both $<12 \%$ ) for all the major lipid classes and subclasses (Supplemental Figure S2 and Supplemental Tables S3, S4; https://doi.org/10.3168/jds .2017-13977). For validation of our lipidomic workflow, we compared results of the summed signal intensities of individual fatty acids with the total fatty acid results using the biochemical colorimetric method. As shown in Supplemental Figure S3 (https://doi.org/10.3168/ jds.2017-13977), results for summed signal intensities of individual fatty acids using MS were in close agreement with the fatty acid results using the biochemical colorimetric method for the 68 examined samples, as exemplified by the Pearson correlation $\left(\mathrm{r}=0.91\right.$ and $\mathrm{R}^{2}$ $=0.84$ ). Further details and the discussion about the analytical workflow are provided in the Supplemental Files (https://doi.org/10.3168/jds.2017-13977).

\section{Serum Metabolite Differences Between CMP and Control Cows}

A total of 2,200 features were detected in both UPLC-MS ${ }^{\mathrm{E}}$ positive and negative modes combined during a 12-min retention time window. We annotated putatively 81 unique molecular metabolites to be 17 proteinogenic AA, 16 AA metabolites, 4 dipeptides, 15 carnitines (Car), 7 bile acids (BA), 6 water-soluble phospholipid (PL) metabolites, 6 carbohydrates, 4 nucleotides and nucleotide metabolites, 3 cholesterol and ChoE, 2 vitamins, and 1 sphingosine metabolite (Supplemental Table S6; https://doi.org/10.3168/jds .2017-13977). Linear, supervised multivariate analysis of extracted metabolite features from the negative mode displayed distinct clustering and clear separation of CMP and control cows at each sampling time (Supplemental Figure S4A and File S5; https://doi .org/10.3168/jds.2017-13977).

Of the 81 metabolites, 17, 7, 0, and 1 could correctly classify for future $\mathrm{CM}$ of cows (AUC-ROC $=1$ ) at $-21,-14,-7$, and $0 \mathrm{~d}$, respectively (Table 1). Each metabolite was higher before calving in CMP versus control cows, and median fold changes of each metabolite decreased as calving approached. Each of the 7 metabolites, $N$-methylethanolamine phosphate, choline, phosphorylcholine, free carnitine, trimethyl lysine, tyrosine, and proline, that could correctly classify for future CM at $-14 \mathrm{~d}$ could also do so at $-21 \mathrm{~d}$. Summed signal intensities of AA metabolites, water-soluble PL metabolites, and carnitines could correctly classify for future $\mathrm{CM}$ of cows $(\mathrm{AUC}-\mathrm{ROC}=1)$ at both -21 and $-14 \mathrm{~d}$.

Significant $(P<0.05)$ group differences between CMP and control cows were observed at all 4 sampling time for 2 metabolites, 3'-sialyllactose (3'-SL; Figure 2A) and glycodeoxycholic acid (Table 1); the presence of both was confirmed by comparison with commercial standards and fragmentation patterns (Supplemental Figure S5 for 3'SL; https://doi.org/10.3168/jds.2017 -13977). Five additional metabolites, lactose, betaine, free carnitine, Car C3:0, Car C18:1, and proline, differed at $-21,-14$, and $-7 \mathrm{~d}$ (all higher in CMP vs. control cows), as did summed signal intensities of AA metabolites (Figure 2B), carnitines (Figure 2C), and water-soluble PL metabolites (Figure 2D). Three additional metabolites, hippuric acid, taurocholic acid, and glycocholic acid, were higher in CMP versus control cows at -21 and $-14 \mathrm{~d}$ and lower at $0 \mathrm{~d}$, as were summed signal intensities of conjugated BA (Figure $2 \mathrm{E}$ ). The largest fold changes before calving among all metabolites and lipids were observed for the mammary gland-derived carbohydrates 3'-SL and lactose (Table 1 ), which showed similar temporal group differences (Figure 2A).

Before calving, 5 metabolite families, AA metabolites, proteogenic AA (Figure 2B), water-soluble PL, carnitines, and conjugated BA (see Supplemental Figures S6, S7, and S8 for PL, carnitines, and conjugated BA, respectively; https://doi.org/10.3168/jds.2017-13977), had over half of the individual metabolites with higher signal intensities in CMP versus control cows: 14, 11, and 2 of 16 identified AA metabolites; 5,6 , and 4 of 6 identified water-soluble PL; 9, 9, and 7 of 15 identified carnitines; and 4, 3, and 1 of 5 identified conjugated BA were higher in CMP versus control cows at -21 , -14 , and $-7 \mathrm{~d}$, respectively. The largest group differences of individual metabolites were observed at $-21 \mathrm{~d}$ and the smallest at $-7 \mathrm{~d}$.

At calving, serum metabolites were either lower in CMP versus control cows or similar (Supplemental Table S7; https://doi.org/10.3168/jds.2017-13977). Of the 14 metabolites that differed, 11 were lower. Those included all 5 identified conjugated BA individually (Supplemental Figure S8) as well as their summed signal intensities (Figure 2E). The remaining metabolites that differed at calving in CMP versus control cows were distributed over all metabolite families: lower were 1 conjugated BA precursor (glycine), 1 water-soluble PL metabolite (glyceryl-phosphoethanolamine), 1 AA metabolite carrier (Car C5:0), and 2 AA metabolites (hippuric acid, indoxyl sulfate) and higher were $1 \mathrm{AA}$ (histidine), 1 AA metabolite (ketoleucine) and 3'-SL. 


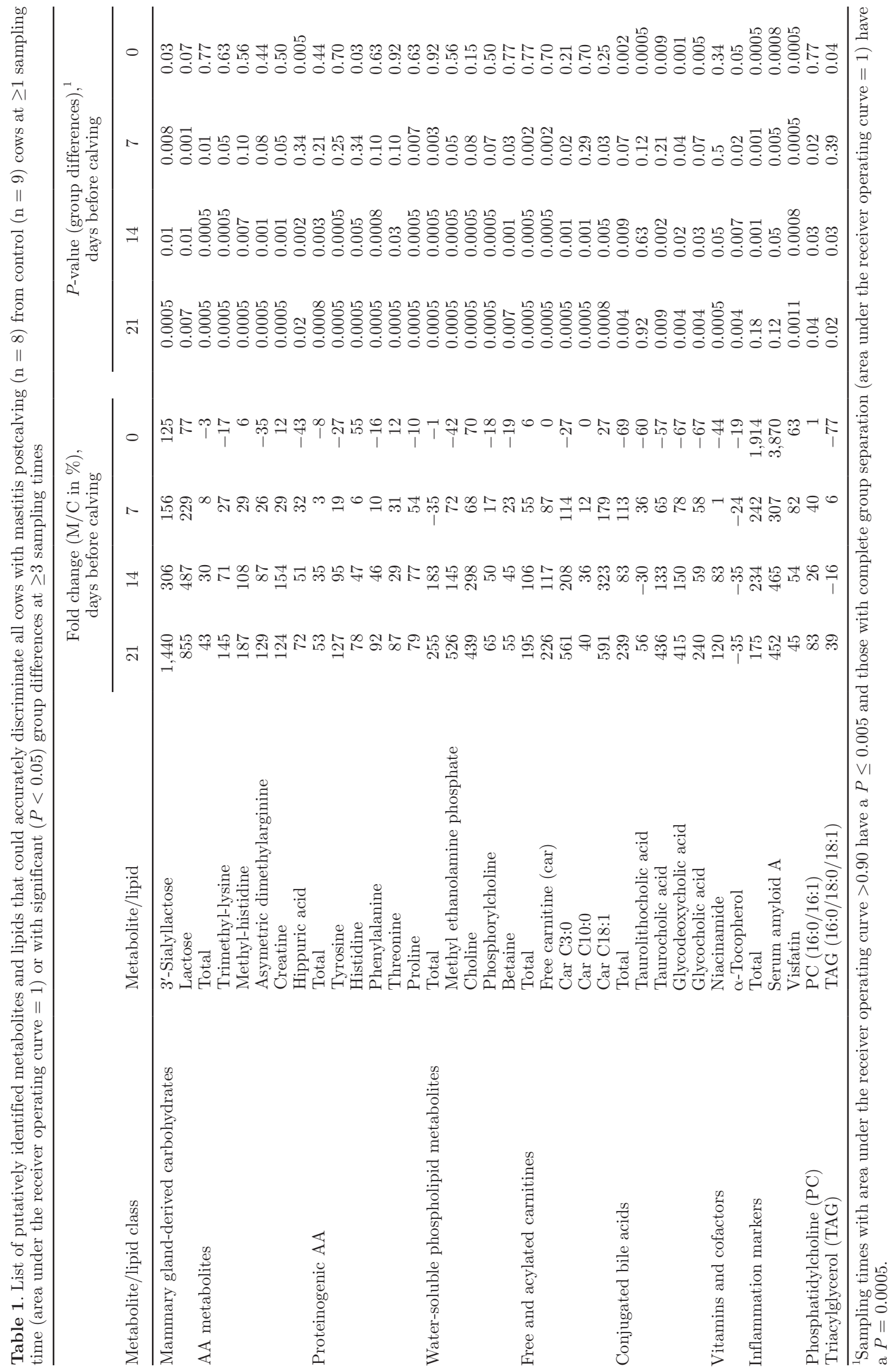



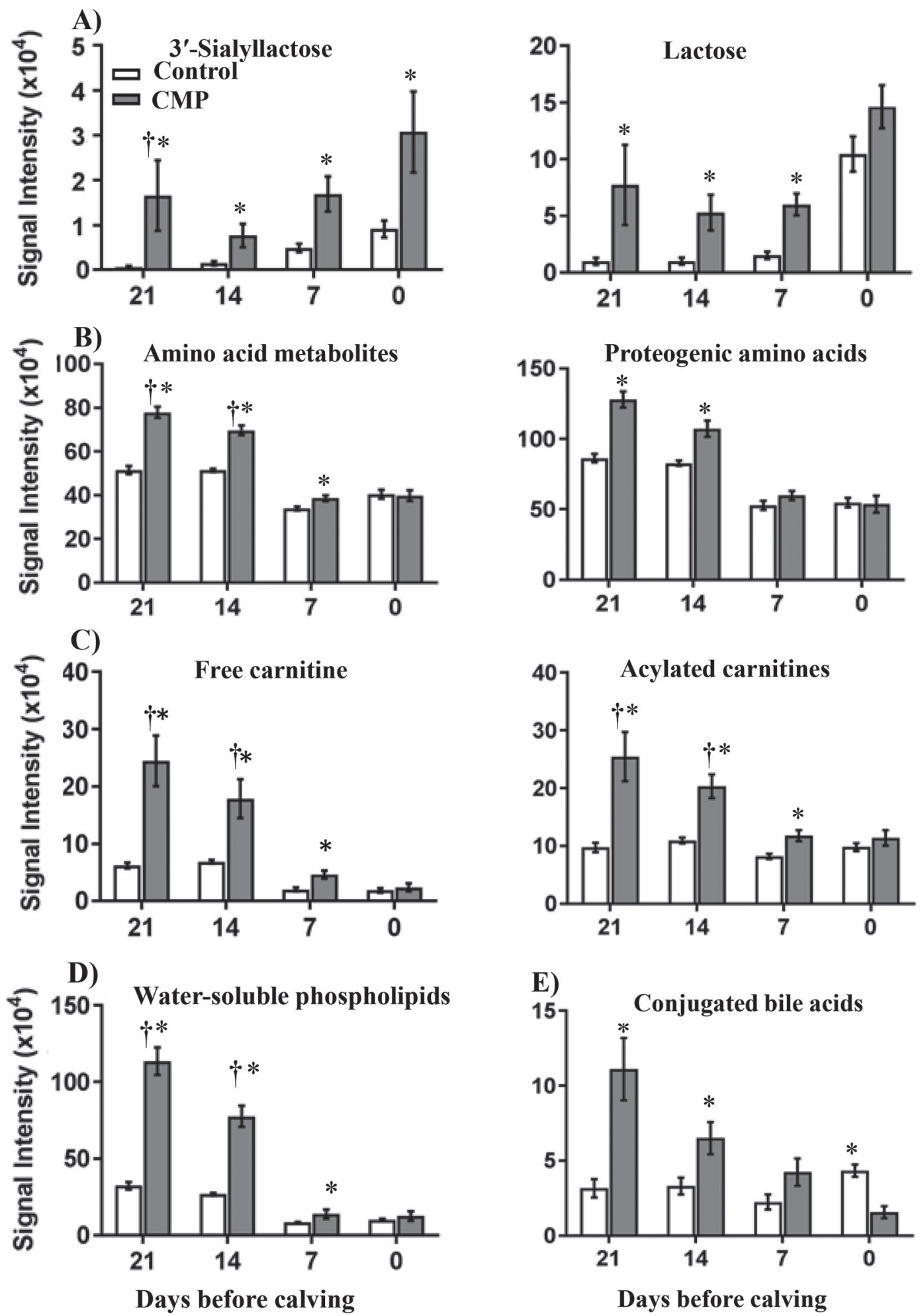

Figure 2. Summed serum signal intensities (mean \pm SEM) of (A) 3'-sialyllactose and lactose, (B) AA metabolites and proteogenic AA, (C) free and acylated carnitines, (D) water-soluble phospholipids, and (E) conjugated bile acids in serum of cows diagnosed with mastitis postcalving (CMP; $\mathrm{n}=8)$ and control $(\mathrm{n}=9)$ cows at $21,14,7$, and $0 \mathrm{~d}$ before calving. Crosses $(\dagger)$ indicate complete group separation (area under the curve-receiver operating characteristics $=1)$, and asterisks indicate significant $(P<0.05)$ group differences between CMP and control cows using Wilcoxon rank sum test. 


\section{Serum Lipid Differences Between CMP Versus Control Cows}

A total of more than 2,200 features were detected in both UPLC-MS ${ }^{\mathrm{E}}$ positive and negative modes combined during a 15-min retention time window. We annotated putatively 204 individual molecular lipids to be 41 phosphocholine (PC), 24 phosphoethanolamine (PE), 10 phosphoinositol (PI), 21 phosphoserine (PS), 19 sphingomyelin (SM), 12 lysophosphocholine (LPC), 10 lysophosphoethanolamine (LPE), 1 lysophosphoinositol (LPI), 2 diacylglycerols (DG), 23 TAG, 4 ChoE, 12 ceramides (Cer), and 24 fatty acids (Supplemental Table S8; https://doi.org/10.3168/jds .2017-13977). Supervised, linear, multivariate analysis on the extracted lipidomic features from the negative mode depicts distinguishable clustering of control and CMP cows at each sampling time (Supplemental Figure S4B and File S5; https://doi.org/10.3168/jds.2017 -13977). However, none of the individual lipids or lipid families could correctly classify for future CM status of cows (AUC-ROC $=1$ ). The best lipid discriminator was the relative proportion of free on total fatty acids (free/total fatty acids\%), which could correctly classify at calving for future CM status of cows except for the only CMP cow that had normal SCC at the onset of lactation (Figure 3A). The only 2 lipids that had significant differences in signaling intensities at $\geq 3$ sampling times were $\mathrm{PC}(16: 0 / 16: 1)$ and TAG (16:0/18:0/18:1; Table 1).

At $-21 \mathrm{~d}, 23$ lipids differed (all $P<0.05$ ) between CMP and control cows. Summed signal intensities of esterified fatty acids (sum of fatty acids of Cer, TAG, DG, LPC, LPE, LPI, PC, PE, PI, PS, and SM; +23\%) were higher $(P=0.04)$ in CMP versus control cows (Figure $3 \mathrm{~A}$ ); this was primarily due to higher signal intensities of the most abundant esterified fatty acids, PC $(+35 \%$; Figure 3B) and UFA containing TAG $(+24 \%$; Figure 3B). Moreover, the ratio of 18:1 to 18:0 fatty acids (C18 desaturase index) in TAG was higher $(+57 \%)$ in CMP versus control cows. In contrast, summed signal intensities of very long chain SFA (20:0 to $28: 0 ;-45 \%)$ and n-3 PUFA $(18: 3,20: 5,22: 5 ;-33 \%)$ were lower (all $P<0.05)$ in CMP versus control cows at $-21 \mathrm{~d}$ before calving (Figure 3C). As calving approached, less lipid ions differed between CMP versus control cows (11 at $-14 \mathrm{~d}$ and 12 at $-7 \mathrm{~d}$ ).

At calving, the largest number (i.e., 59) of lipids differed between CMP versus control cows (Supplemental Table S9; https://doi.org/10.3168/jds.2017-13977). Summed signal intensities of fatty acids $(+121 \%)$ were higher $(P=0.01)$ in CMP versus control cows (Figure $3 \mathrm{~A}$ ), which was primarily due to the higher signal intensities of the most abundant fatty acids, long-chain SFA
$(+112 \% ; P=0.02)$, and MUFA $(+182 \% ; P=0.005)$ (Figure 3C). Signal intensities of each identified longchain fatty acid and MUFA were higher in CMP versus control cows (Supplemental Figure S9; https://doi .org/10.3168/jds.2017-13977). In contrast to long-chain fatty acids, summed signal intensities of very long chain SFA $(-23 \% ; P=0.007), \mathrm{PC}(-19 \% ; P=0.03$; Figure $3 \mathrm{~B})$, PI $(-26 \% ; P=0.02$; Figure 3B), 18:0-containing TAG $(-53 \% ; P=0.02)$, monohexosyl Cer $(-29 \% ; P=$ 0.04 ), and summed signal intensities of UFA-containing LPC $(-46 \% ; P=0.007$; Supplemental Figure S10; https://doi.org/10.3168/jds.2017-13977), LPE (-42\%; $P=0.01$; Supplemental Figure S11; https://doi.org/10 $.3168 /$ jds.2017-13977), and ChoE $(-41 \% ; P=0.004)$ were lower in CMP versus control cows (Figure 3D). Moreover, the ratio of UFA-containing LPE to PE $(-65 \% ; P<0.0001$, complete group separation) and the ratio of UFA-containing LPC to PC $(-17 \% ; P<$ 0.03) were lower in CMP versus control cows, whereas the ratio of 18:1 to 18:0 FA (C18 desaturase index) in TAG was higher $(+274 \% ; P<0.007)$.

\section{Serum Biochemical Differences Between CMP Versus Control Cows}

For biochemical analysis, we quantified serum concentrations of markers of acute and chronic inflammation (haptoglobin, SAA, tumor necrosis factor- $\alpha$, visfatin; Supplemental Figure S12A, https://doi.org/10.3168/ jds.2017-13977), lipoprotein metabolism ( $\alpha$-tocopherol, cholesterol; Supplemental Figure S12B), energy status (fatty acids, BHB, glucose, urea N; Supplemental Figure $\mathrm{S} 12 \mathrm{C}$ ), and mineral status (calcium, magnesium, phosphorus; Supplemental Figure S12D). In addition, we measured BCS at each sampling time (Supplemental Figure S13; https://doi.org/10.3168/jds.2017-13977). The PLS-DA score plots showed distinct clustering of CMP and control cows at each sampling time except at $14 \mathrm{~d}$ before calving (Supplemental Figure S4C and File S5; https://doi.org/10.3168/jds.2017-13977).

Separation between CMP and control cows was driven by SAA, visfatin, and $\alpha$-tocopherol. Whereas markers of inflammation were higher in CMP versus control cows throughout the sampling period, serum concentrations of $\alpha$-tocopherol were lower (Table 1). Complete group separation were observed for visfatin at -7 and 0 d. Significant group differences at all 4 sampling times were observed for visfatin and $\alpha$-tocopherol and at the last 3 sampling times for SAA and summed concentrations of inflammatory markers (Figure 4).

Similar to the results obtained with MS, serum concentrations of total fatty acids were higher $(P \leq 0.05)$ in CMP versus control cows at $-7(+139 \%)$ and $0 \mathrm{~d}$ $(+178 \%)$. Higher serum concentrations of haptoglobin 

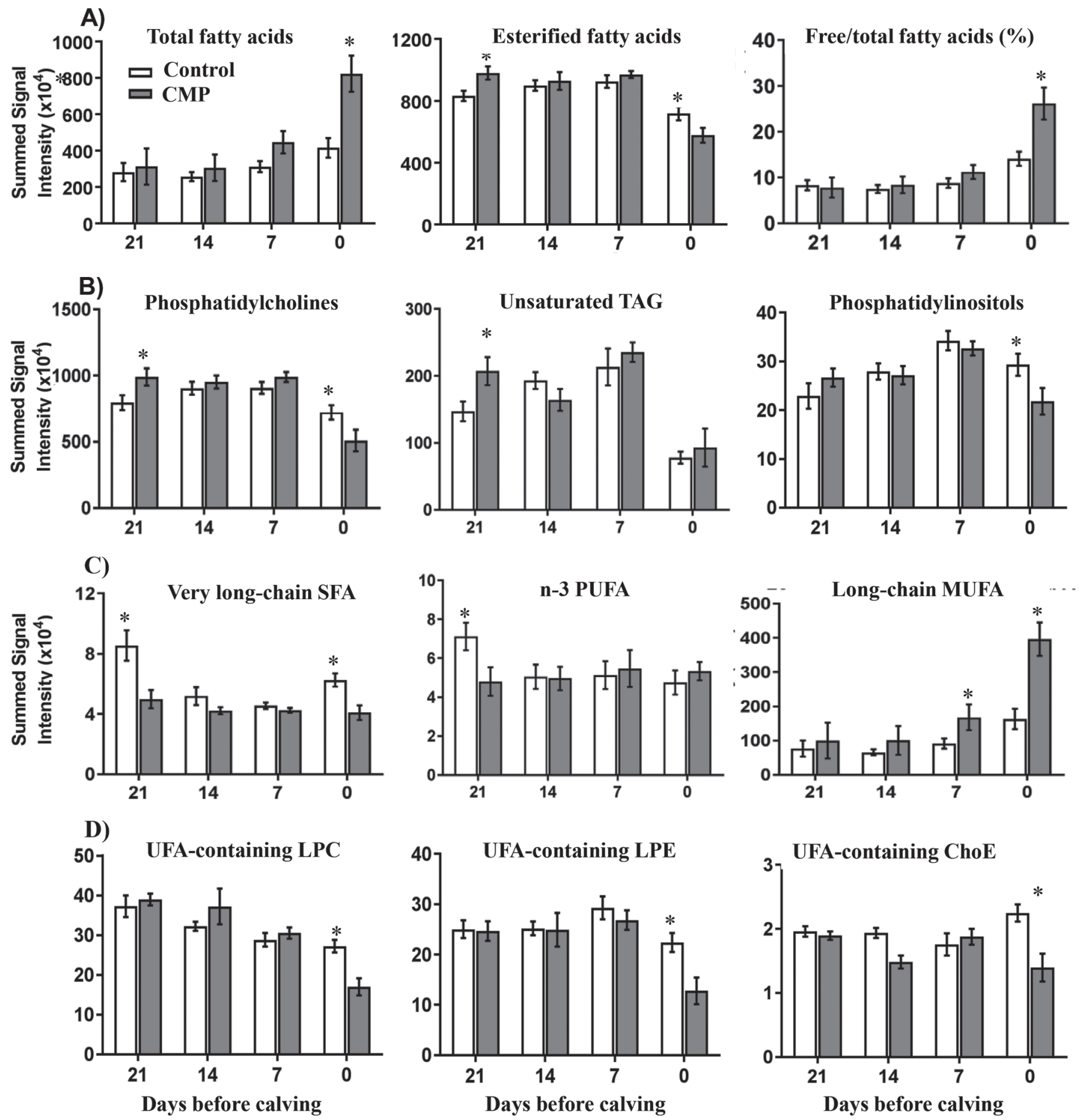

Figure 3. Summed serum signal intensities (mean \pm SEM) of total (A) free fatty acids, esterified fatty acids, and proportion of free on total fatty acids, (B) phosphatidylcholine, unsaturated triacylglycerol (TAG), and phosphatidylinositols, (C) very long chain SFA, n-3 PUFA, and long-chain MUFA, and (D) UFA-containing lysophosphocholine (LPC), lysophosphoethanolamine (LPE), and cholesteryl esters in serum of cows diagnosed with mastitis postcalving $(\mathrm{CMP} ; \mathrm{n}=8)$ and control $(\mathrm{n}=9)$ cows at $21,14,7$, and 0 d before calving. Asterisks indicate significant $(P \leq 0.05)$ group differences between CMP and control cows using Wilcoxon rank sum test.

were not observed in CMP versus control cows until after calving ( $0 \mathrm{~d}=+251 \%$; results after calving not shown). Other indicators and indicator families showed no or limited ability to predict $\mathrm{CM}$ and differed $(P$ $\leq 0.05$ ) between CMP and Control cows at only 1 (glucose, phosphorus) or 0 sampling times (BHB, cal- 

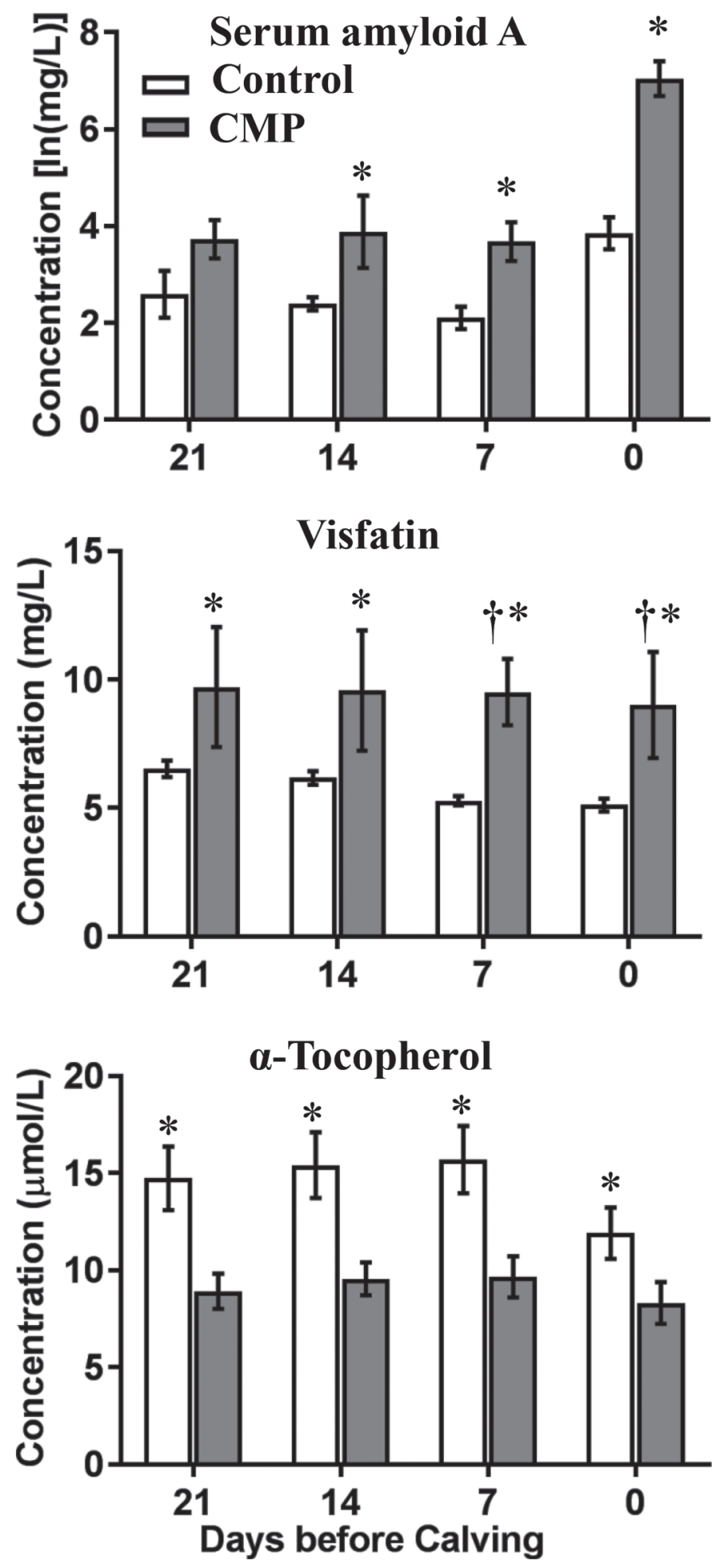

Figure 4. Serum concentrations (mean \pm SEM) of serum amyloid $A$, visfatin, and $\alpha$-tocopherol measured using biochemical analysis at $21,14,7$, and $0 \mathrm{~d}$ before calving. Crosses $(\dagger)$ indicated complete group separation (area under the curve-receiver operating characteristics $=$ $1)$, and asterisks show significant $(P \leq 0.05)$ differences between cows diagnosed with mastitis postcalving $(\overline{\mathrm{C} M P} ; \mathrm{n}=8)$ and control $(\mathrm{n}=9)$ cows using Wilcoxon rank sum test. cium, cholesterol, magnesium, urea $\mathrm{N}$, tumor necrosis factor- $\alpha$, BCS).

\section{Temporal Serum Metabolite and Lipid Changes During the Prepartal Transition Period}

Temporal serum metabolite changes during the prepartal transition period were visualized using SOM in GEDI. For metabolomics results, GEDI grouped carnitines, AA and their metabolites, and conjugated BA in distinct and separate tiles (Figure 5A). Temporal metabolite changes differed between metabolite families as well as between CMP and control cows. Larger decreases in summed signal intensities were observed in CMP versus control cows for AA ( -60 vs. $-34 \%$ Figure $2 \mathrm{~B}$ ) and AA metabolites ( -46 vs. $-22 \%$; Figure 2B), free Car (-91 vs. $-69 \%$; Figure 2C), and watersoluble PL ( -91 vs. $-69 \%$; Figure 2D), all of which primarily decreased between -14 and $-7 \mathrm{~d}$. Summed signal intensities of acylated carnitines (control cows $=+6 \%, P=0.56 ; \mathrm{CMP}$ cows $=-52 \%, P<0.0001$, primary decrease between -14 and $-7 \mathrm{~d}$; Figure $2 \mathrm{C}$ ) and conjugated BA (control cows $=+37 \%, P=0.33$; CMP cows $=-87 \%, P<0.0001$, consistent decrease throughout; Figure 2E) only decreased in CMP cows.

For the lipidomics results, GEDI grouped fatty acids, TAG, PC, LPC and LPE, and PE in distinct and separate tiles (Figure 5B). Temporal metabolite changes differed between metabolite families as well as between CMP and control cows. During the prepartal transition period, cows transitioned from lipid synthesis to lipid catabolism. Relative abundance of fatty acids increased exponentially starting $-7 \mathrm{~d}$, whereas the relative abundance of esterified fatty acids, including TAG, LPC and LPE, and PC, decreased until calving. Larger temporal changes were observed in CMP versus control cows; the proportion of free on total fatty acids (free/total fatty acids\%) increased greater in CMP versus control cows $(+333$ vs. $+82 \% ; P=0.02$; Figure $3 \mathrm{~A})$, as summed signal intensities of fatty acids (CMP vs. control cows: +338 vs. $+89 \%$; Figure $3 \mathrm{~A})$ and summed signal intensities of LPC ( -59 vs. $-39 \%), \mathrm{PC}(-49$ vs. $-15 \%), \mathrm{PE}$ $(-24$ vs. $+5 \%)$, and PI $(-26 \%$ vs. $+36 \%)$ changed in opposite directions, the change being significant only in CMP cows. Summed signal intensities of PS, LPE, SM, monohexosyl Cer, and ChoE as well as cholesterol decreased similarly in both groups during the prepartal transition period.

\section{Integrative Overview of Dynamic Changes During the Prepartal Transition Period}

Dynamic changes of metabolites, lipids, and inflammatory markers during the prepartal transition period 
are linked and integrated in Figure 6 to provide a schematic representation of our study results. At -21 d (Figure 6A), serum markers of muscle protein breakdown (AA and their metabolites, odd short-chain acyl carnitines), muscle and liver FA oxidation (long-chain acyl carnitines), chronic inflammation (visfatin), liver choline metabolism (water-soluble PL metabolites), mammary gland development (mammary gland-derived carbohydrates), and liver lipid synthesis, packaging, secretion, and recycling (conjugated BA, PL, TAG) were higher in CMP versus control cows. Whereas group differences between serum metabolites decreased until calving, except for markers of early mammary gland development, signal intensities of serum markers of acute inflammation (SAA) and adipocyte TAG breakdown (long-chain saturated and monounsaturated fatty

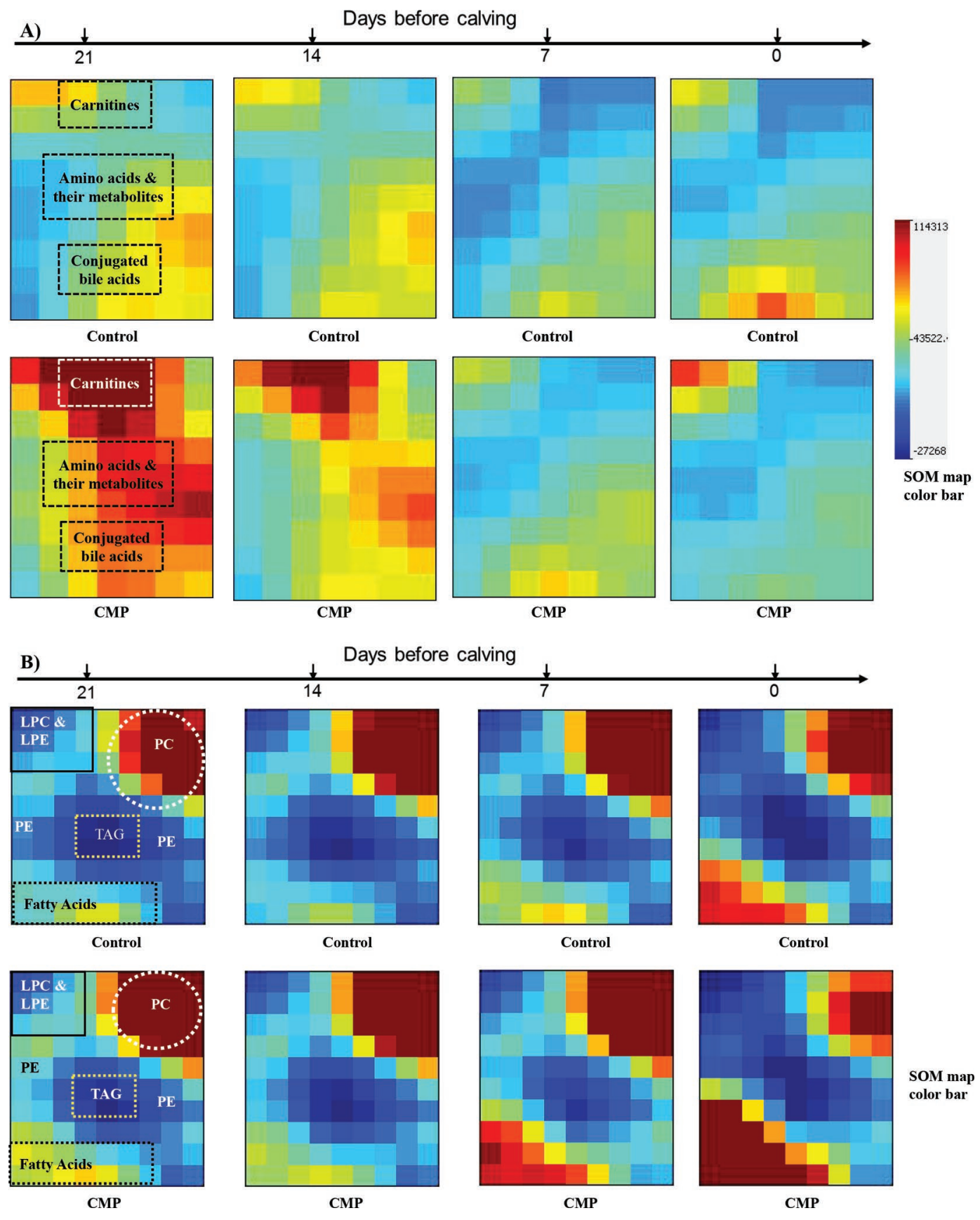

Figure 5. Self-organizing maps (SOM) of (A) metabolomic and (B) lipidomic data of cows' serum samples diagnosed with clinical mastitis postcalving (CMP) and control cows at 21, 14, 7, and $0 \mathrm{~d}$ before calving are visualized in Gene Expression Dynamics Inspector (GEDI; https:// apps.childrenshospital.org/clinical/research/ingber/GEDI/gedihome.htm). LPC = lysophosphocholine; LPE = lysophosphoethanolamine; PE $=$ phosphotidylethanolamine. Color version available online. 
acids) increased in CMP cows starting at $-14 \mathrm{~d}$ but not in control cows before calving. At calving (Figure $6 \mathrm{~B})$, serum markers of acute and chronic inflammation (SAA, visfatin) and adipocyte TAG breakdown catabolism (long-chain SFA and MUFA) were higher and markers of liver lipid synthesis, packaging, secretion, and recycling as well as intestinal lipid absorption and synthesis (esterified fatty acids, PC, PI, C18:0 containing TAG, unsaturated LPC, LPE, and ChoE, and conjugated $\mathrm{BA}$ ) were lower in $\mathrm{CMP}$ versus control cows at calving.

\section{DISCUSSION}

The last 3 wk before calving, also called prepartal transition period, is marked by homeorhetic metabolomic and lipidomic adaptations for the upcoming parturition and lactation, which are orchestrated by hormonal changes (Drackley, 1999; Ingvartsen, 2006). Reasons for these adaptations include exponentially increasing energy, AA, and lipid requirements for the growing fetus and expanding mammary gland; and accumulation of minerals and vitamins, growth factors, antioxidants, immunoglobulins, and bactericidal compounds in the colostrum to strengthen immune-response of the immune-naïve neonate. In addition, fetus size may limit rumen size and, thus, feed intake. During the transition period, immunosuppression results in increased susceptibility to infectious diseases such as CM. Given this physiological background, the objective of our study was to identify predictive indicators that precede $\mathrm{CM}$ in dairy cows.

\section{Metabotype Differences Between CMP and Control Cows at -21 and -14 Days}

Elevated signal intensities of multiple serum AA and AA metabolites and carnitines were observed in CMP cows at -21 and $-14 \mathrm{~d}$. Carnitine and AA metabolism are linked, as the breakdown of endogenous proteins containing trimethyl lysine residues is recognized as the starting point for free carnitine synthesis (Servillo et al., 2014). Acylated carnitines play important roles in both AA oxidation, specifically short-chain odd acylcarnitines (Car C3:0 and CarC 5:0), and fatty acid $\beta$-oxidation, specifically long-chain even acylcarnitines in muscle and liver (Koves et al., 2008). Medium-chain acylcarnitines (Car C10:0) indicate incomplete fatty acid $\beta$-oxidation when fatty acid availability exceeds the amount that can be oxidized in the mitochondria. Elevated AA and AA metabolites and carnitines have been reported in previous research at 8 or 4 wk prepartum in cows diagnosed postpartum with various diseases, including SCM (Hailemariam et al., 2014;
Dervishi et al., 2017; Zhang et al., 2017). It was proposed that inflammation caused the observed metabolic changes; for example, elevated concentrations of serum haptoglobin, tumor necrosis factor- $\alpha$, and IL-1 preceded SCM diagnosis 4 wk prepartum (Dervishi et al., 2015).

To evaluate whether inflammation associated with infection may have triggered the CMP metabotype observed at $-21 \mathrm{~d}$, we measured serum markers of chronic (visfatin) and acute inflammation (SAA, haptoglobin, tumor necrosis factor- $\alpha$ ). Visfatin, but not markers of acute inflammation, were elevated in CMP versus control cows. We checked also for previous SCC and mastitis events of CMP cows, and the results indicated that CMP cows were most likely free of mammary infections at $-21 \mathrm{~d}$. Thus, chronic inflammation may precede AA and FA catabolism, which may cause those cows to be more susceptible to mammary infections (Sordillo et al., 2009).

One question that arises is whether the elevated AA and FA catabolism can be explained by decreased feed intake rather than inflammation. The answer is not straightforward, as a synergistic interrelation exists between inflammation, decreased feed intake, and elevated AA and FA catabolism. Inflammation-associated cytokine and hormone release decreases feed intake and increases protein and lipid catabolism in liver and muscle; however, the reverse is also true (Sordillo et al., 2009; Kuhla et al., 2011). Thus, the question becomes what happened first: inflammation, the AA and FA catabolic metabolic phenotype (i.e., metabotype), or low feed intake. We cannot answer this question, as chronic inflammation and the AA and FA catabolic metabotype both were evident at $-21 \mathrm{~d}$. A limitation for the interpretation of our data is that we did not measure feed intake; however, we can use fatty acids, $\mathrm{BHB}$, and BCS as indicator of energy status (Moyes et al., 2013), and none differed between CMP and control cows at -21 and $-14 \mathrm{~d}$. We propose that cows develop a chronic inflammatory metabotype (i.e., "inflammaging") as they age, similar to what has been proposed in literature on humans (Franceschi et al., 2007). Besides chronic inflammation, hallmarks of inflammaging are progressive mitochondrial dysfunction, as indicated by elevated AA and FA catabolism, and increased reactive oxygen species accumulation and cellular damage (López-Otín et al., 2013), which may be the cause of low serum concentrations of $\alpha$-tocopherol in CMP cows. In support of our proposed mechanism, Huber et al. (2016) observed an inflammatory metabotype with mitochondrial dysfunction in early-lactation dairy cows that later failed to conceive, which was not linked to decreased feed intake.

Whereas Huber et al. (2016) and other recent studies used a targeted metabolomics approach, our untargeted 
A)

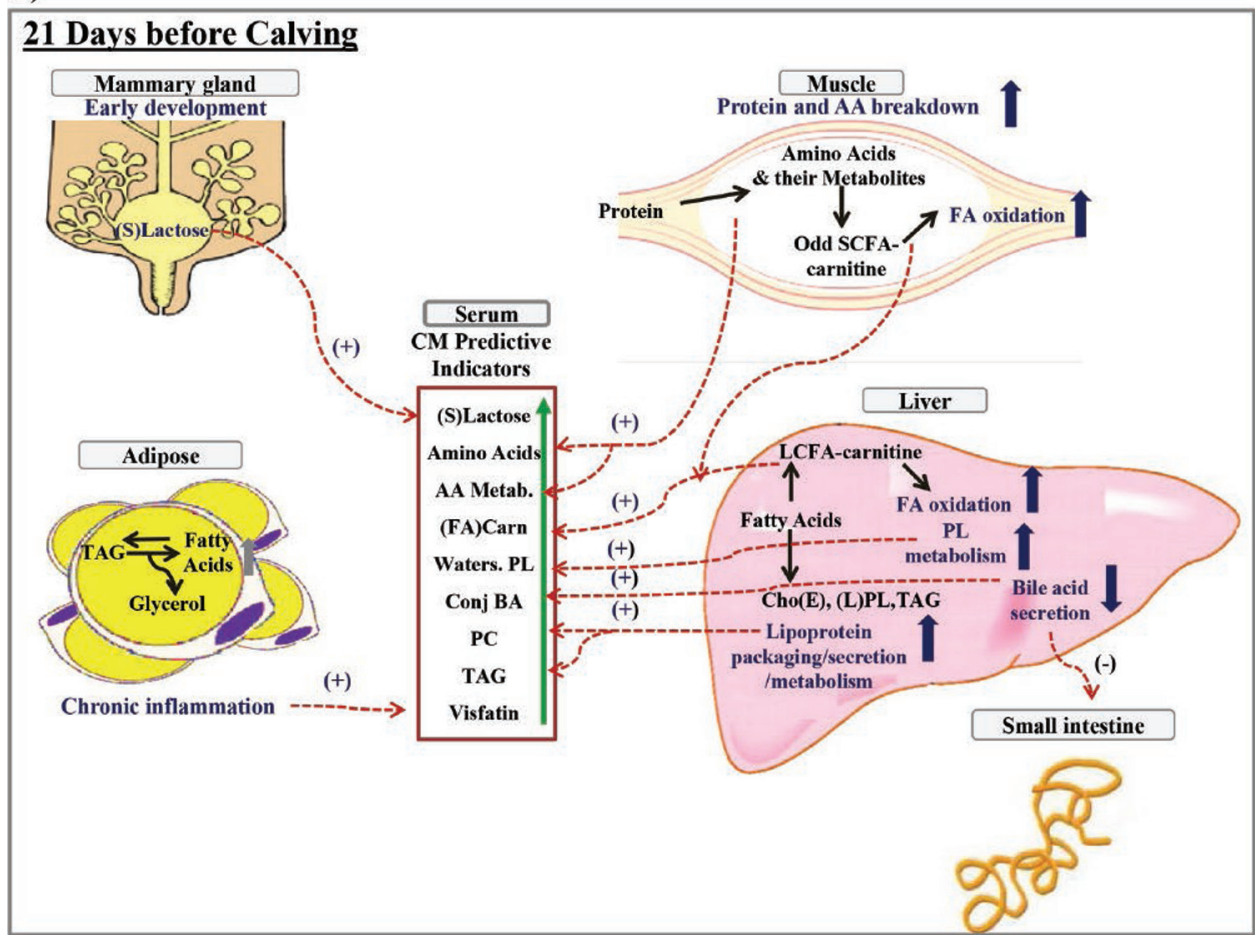

B)

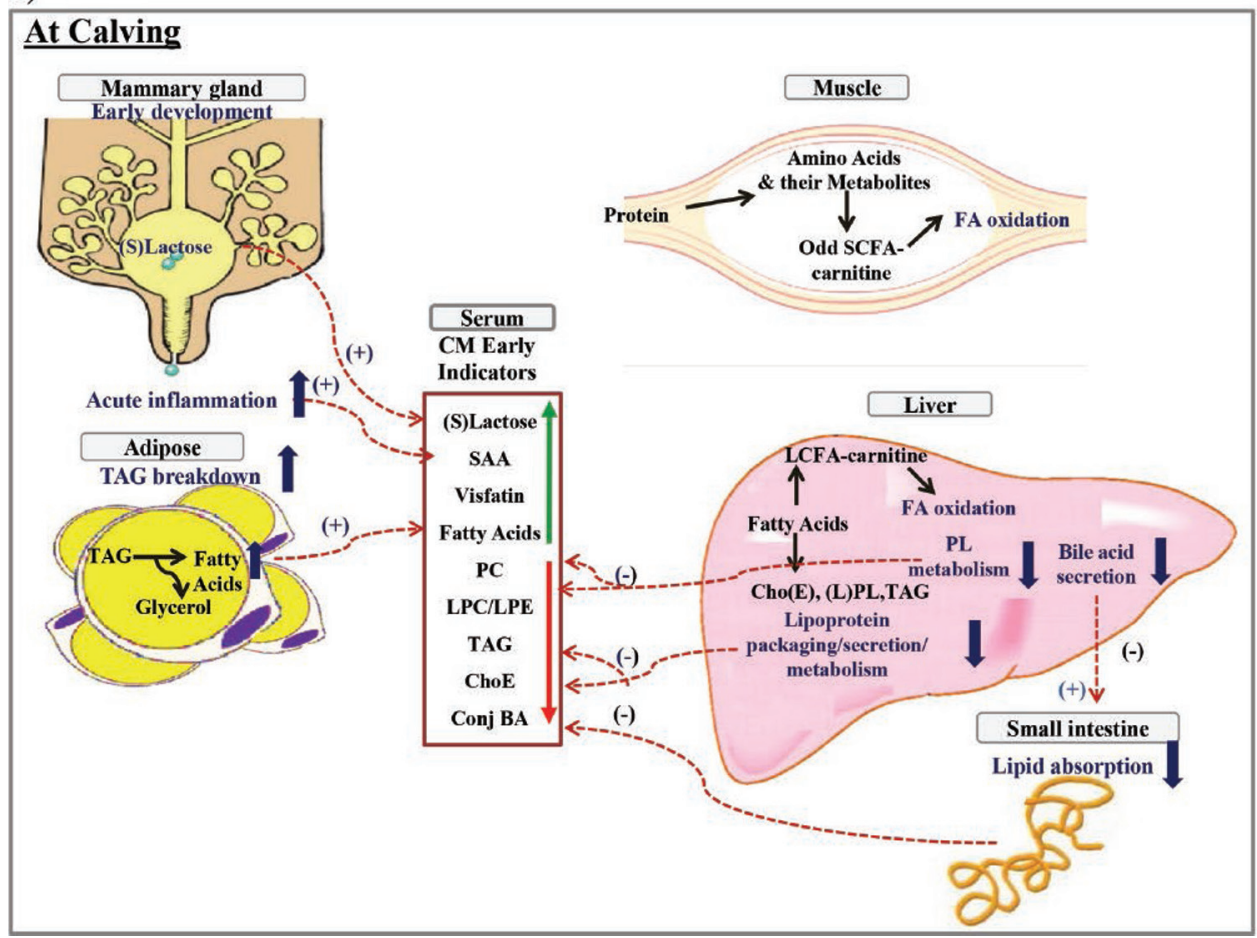

Figure 6. Schematic representation of proposed cross-talk between mammary gland, adipose tissue, muscle, liver, small intestine, and pathogenic bacteria in cows diagnosed with mastitis postcalving (CMP) versus control cows (A) at $21 \mathrm{~d}$ before calving and (B) at calving. Proposed processes and their differences between CMP versus control cows in tissue are in dark bold arrows. Dashed lines with plus signs $(+)$ indicate higher and minus signs $(-)$ indicate lower signal intensities in serum in CMP versus control cows. ChoE $=$ cholesteryl esters; ConjBA $=$ conjugated bile acids; FA (Carn) $=$ free and acylated carnitines; FA $=$ fatty acid; LCFA-carnitine $=$ long-chain acylated carnitines; LPL $=$ lysophospholipids (LPE and LPC); PL = phospholipids [phosphotidylcholines (PC) and phosphotidylethanolamine (PE)]; Odd SCFA-carnitine $=$ short-chain odd acylated carnitines; $\mathrm{SAA}=$ serum amyloid $\mathrm{A} ;(\mathrm{S})$ lactose $=3$ '-sialyllactose and lactose; TAG = triacylglycerol; and watersoluble PL $=$ water soluble phospholipids. Color version available online. 
metabolomic and lipidomic study allowed us to look at a broader number of metabolite and lipid families (e.g., BA and water-soluble PL). At -21 and $-14 \mathrm{~d}$, elevated levels of conjugated BA were observed in CMP cows. Our results for conjugated BA are similar to those observed in human pregnancy-associated cholestasis, in which pregnancy hormones stop or slow down bile flow, resulting in a build-up of BA in the liver that spills in the blood stream (Lammert et al., 2000; Geenes and Williamson, 2009). Our observed elevated PC levels at $-21 \mathrm{~d}$ may be related to the elevated conjugated BA levels indicating pregnancy-associated cholestasis. Decreased bile flow, as indicated by elevated conjugated BA concentrations, may interfere with hepatic removal of pathogens and their toxic products. Future studies are warranted to examine whether pregnancyassociated cholestasis is a health problem in dairy cows.

At -21 and $-14 \mathrm{~d}$, nearly all water-soluble PL metabolites were elevated in CMP versus control cows. In humans, pregnancy (i.e., increased choline transfer into the fetus) as well as various diseases (e.g., coronary heart disease) are associated with elevated circulating choline concentrations (Danne et al., 2007; Yan et al., 2012). This is likely because the high needs for methyl groups of fast-growing and metabolizing tissues (i.e., nucleotide synthesis, methylation) and high choline and betaine excretion result in elevated choline metabolism (Yan et al., 2012; Ducker and Rabinowitz, 2017). Carnitine, AA and AA metabolites, and water-soluble PL metabolites are metabolically linked, as choline via betaine provides methyl groups for carnitine biosynthesis (Griffith, 1987; Zhou et al., 2017), which is essential for AA and fatty acid metabolism. Thus, elevated serum levels of water-soluble PL metabolites may indicate both inflammation-associated diseases and elevated AA and fatty acid catabolism in muscle and liver.

One of the most intriguing observations from a physiologic perspective involved summed signal intensities of esterified fatty acids (sum of fatty acids of Cer, DG, LPC, LPE, LPI, PC, PE, PI, PS, SM, and TAG). At $-21 \mathrm{~d}$, summed signal intensities of esterified fatty acids were higher in CMP versus control cows, which was primarily due to higher signal intensities of the most abundant esterified fatty acid-containing lipid ions, PC and TAG containing 18:1, 18:2, or both. Circulating TAG are primarily associated with very low density lipoprotein (VLDL), and the fatty acid composition of TAG mainly reflects hepatic lipid metabolism and secretion (Gray et al., 2013). We interpret our observation as an indication of elevated hepatic lipid biosynthesis and VLDL secretion in CMP versus control cows. Stearoyl-CoA desaturase is the rate-limiting enzyme in MUFA synthesis and critical for both hepatic lipid biosynthesis and VLDL secretion (Stefan et al., 2008).
We observed higher 18:1-to-18:0 fatty acid ratios (i.e., C18 desaturase index) in TAG of CMP versus control cows at $-21 \mathrm{~d}$, suggesting greater hepatic stearoyl CoA desaturase activity in CMP versus control cows. In humans, greater hepatic stearoyl CoA desaturase 1 activity is related to more VLDL secretion and less liver fat, but also to increased hepatic lipid synthesis and less hepatic fatty acid oxidation (Stefan et al., 2008; Silbernagel et al., 2012). Thus, CMP cows may differ from control cows in endogenous lipid metabolism (i.e., higher stearoyl CoA desaturase activity).

Summed signal intensities of 2 fatty acid subfamilies, n-3 PUFA and very long chain SFA, were lower in CMP versus control cows at -21 d. Serum n-3 PUFA are known to have anti-inflammatory properties (Tai and Ding, 2010). Lower circulating concentrations of very-long chain SFA, which have important roles in membrane structure and intracellular signaling, are associated with increased risk of chronic diseases in humans (Lee et al., 2015; Malik et al., 2015). We are not aware of studies examining the relation between circulating very long chain SFA, their synthesis and degradation, and bovine diseases, indicating an area of future research.

\section{Consistent Metabotype Differences Between CMP and Control Cows}

The most consistent single discriminant metabolite in our data was $3^{\prime}$-SL, followed by lactose; both metabolites are synthesized exclusively in the mammary gland. Sialyllactose is the smallest acidic oligosaccharide and most abundant oligosaccharide in bovine milk ( $>50 \%$ of oligosaccharides) and occurs in 2 isomeric forms $3{ }^{\prime}$-SL and 6'-SL (Gopal and Gill, 2000); 3'-SL is more abundant in bovine milk than $6^{\prime}-\mathrm{SL}$, which is the major SL in human milk (Nakamura et al., 2003; Tao et al., 2009; ten Bruggencate et al., 2014). We detected and validated the presence of $3^{\prime}$-SL and $6{ }^{\prime}$-SL by comparing the serum samples with commercial standards, and we only detected 3 '-SL in our serum samples. The proposed function of $3^{\prime}-\mathrm{SL}$ and $6^{\prime} \mathrm{SL}$ is to protect calves against infections (Nakamura et al., 2003) and to support innate immunity in newborns (ten Bruggencate et al., 2014); thus, 3'-SL may be increased during an infection. Both 3 '-SL and lactose increased during the prepartal transition period in control cows, whereas their values were already elevated at $-21 \mathrm{~d}$ in CMP cows, indicating earlier mammary gland development in CMP cows. We excluded delayed calving as a potential explanation for earlier mammary gland development because pregnancy length did not significantly differ between CMP and control cows $(P=0.12)$. Elevated 3 '-SL and lactose in serum of CMP cows may indicate 
passive transfer of mammary gland-derived compounds into serum as a result of pathogen-induced damage of the mammary gland epithelial barrier between blood and milk (Mackenzie and Lascelles, 1968). It is intriguing that a mammary gland-derived oligosaccharide in serum may assist in CM prediction, given that several blood-derived metabolites in milk are used as indicators of CM (Viguier et al., 2009). Further studies are required to validate and quantify this proposed metabolite as a robust predictive indicator of $\mathrm{CM}$ and potentially SCM.

Serum visfatin concentrations were elevated and serum $\alpha$-tocopherol concentrations were lower in CMP versus control cows throughout the prepartal transition period. This is similar to what we previously reported in dairy cows that subsequently developed retained placenta and other diseases (Qu et al., 2013, 2014; Fadden and Bobe, 2015), indicating chronic inflammation and depleted antioxidant reserves are potential risk factors of CM.

\section{Metabotype Differences Between CMP and Control Cows at Calving}

Metabotype differences started to change between -14 and $-7 \mathrm{~d}$. A shift occurred from elevated AA and fatty acid oxidation to increased adipose lipolysis in CMP cows, which may relate to the decrease in carnitine levels and depleted carnitine stores for fatty acid and AA oxidation. We propose that the shift is the result of an acute infection, as indicated by increased SAA concentrations in CMP cows, suggesting that during this time period CMP cows became infected with CM. The SAA increase preceded the increase in long-chain SFA and MUFA, which are fatty acids enriched in adipose tissue (Rukkwamsuk et al., 2000), indicating that acute inflammation most likely caused adipose lipolysis. This sequence of events has been reported in metabolomics studies for other postpartum diseases (Hailemariam et al., 2014; Dervishi et al., 2015, 2017).

Novel findings are the lower serum conjugated BA levels in CMP versus control cows directly after calving, which may indicate decreased intestinal lipid absorption and bile recycling postpartum. This could also explain the lower PL levels of CMP versus control cows at calving. Lower serum BA levels may also explain, in part, the higher acute phase protein concentrations in CMP versus control cows after calving, because lower $\mathrm{BA}$ concentrations may result via less farnesoid $\mathrm{X}$ receptor activation in more proinflammatory cytokine synthesis and inflammation (Wang et al., 2008).

Also novel were the lower signal intensities of esterified fatty acids, PC, PI, C18:0-containing TAG, unsaturated $\mathrm{LPC}$, LPE, and ChoE in CMP versus control cows at calving. As a consequence, the proportion of free on total fatty acids (free/total fatty acids\%) was 2 -fold higher in CMP versus control cows with complete separation of groups except for the only CMP cow that was without disease directly after calving. This makes biological sense, as free on total fatty acid proportion accounts not only for the increased fatty acid release from adipose tissue but also for the decreased esterified fatty acids release from the liver in sick cows. Inadequate PC and PI availability has been implicated in decreased hepatic lipoprotein assembly and lipid secretion in transition dairy cows (Bobe et al., 2004). Serum ChoE are synthesized in serum highdensity lipoproteins by transacylation of $\mathrm{PC}$, a reaction that is inhibited by inflammation (Kontush et al., 2013; Kessler et al., 2014). Our results are consistent with a cytokine-induced decrease in hepatic lipoprotein secretion in response to an acute mammary infection (Gruys et al., 2005; Bannerman et al., 2009). Thus, we interpreted our observations as indication of lower hepatic lipid biosynthesis or secretion in CMP versus control cows at calving in response to acute inflammation and lower intestinal lipid absorption.

Noteworthy is that greater decreases with calving and in CMP versus control cows were observed in fully or partly deacylated PL metabolites than in PL itself. As a result, LPC-to-PC ratio and LPE-to-PE ratio were lower in CMP versus control cows at calving. The results suggest that phospholipase or acyltransferase activity of the Lands cycle may be affected by mastitis infections. In support, pathogenic bacteria and infection have been reported to inhibit phospholipase activity (Hailemariam et al., 2014). In humans, the LPC-to-PC ratio has been proposed as inflammation marker; for example, lower LPC-to-PC ratios were associated with increased risk of mortality in sepsis patients (Drobnik et al., 2003). Thus, decreases in LPC-to-PC ratio and LPE-to-PE ratio may indicate an acute CM infection in dairy cows, as pathogenic bacteria alter PL metabolism.

\section{CONCLUSIONS}

We described and evaluated a novel analytical workflow, suitable for comprehensive global lipidomics and metabolomics profiling of biological specimens (discussed in Supplemental File S6; https://doi.org/ 10.3168/jds.2017-13977). The observed shifts in serum metabolic, lipidomic, and inflammation profile suggest metabolic crosstalk between mammary gland, muscle, adipose tissue, liver, intestine, and pathogenic bacteria to adapt to parturition and an infectious challenge, which will be the objective of future research. Based on the observed shifts, we propose a time sequence of events potentially preceding and following naturally oc- 
curring CM during the close-up period. Early mammary gland development, pregnancy-associated cholestasis, chronic inflammation, elevated choline metabolism, mitochondrial dysfunction, and depleted antioxidant reserves preceded $\mathrm{CM}$ and are potential risk factors of CM. Serum markers of acute inflammation followed by elevated adipocyte lipolysis and decreased lipid and bile synthesis, secretion or recycling may follow CM infection and potentially indicate early subclinical stages of CM. Further studies are required to validate and quantify these proposed risk factors and indicators and determine whether these changes are specific to CM or also occur with other diseases.

\section{ACKNOWLEDGMENTS}

This study was supported by Oregon State University (Corvallis), the Oregon State University Agricultural Research Foundation (Corvallis), the Oregon Beef Council (Portland), Diamond V (Cedar Rapids, IA), and the E.R. Jackman Internship Support Program (Oregon State University). The procurement of the Waters Synapt G2 system was made possible by National Institutes of Health grant S10RR025628 (Bethesda, MD) and funds from the Oregon State University's Research Equipment Reserve Fund. The authors wish to acknowledge the owners and staff of VanBeek Dairy (Monroe, OR) for use of their facilities and their animals; and S. Bledsoe, B. Block, B. Bronson, M. Keller, M. McGuire, P. Ramsing, A. Rudolph, D. Sabedra, C. Sause, C. Shriver-Munsch, M. Swearingen, and E. Zaworski (all at Oregon State University, Corvallis) for their assistance with sample collection and data entry.

\section{REFERENCES}

Bannerman, D. D., M. Rinaldi, B. T. Vinyard, J. Laihia, and L. Leino. 2009. Effects of intramammary infusion of cis-urocanic acid on mastitis-associated inflammation and tissue injury in dairy cow. Am. J. Vet. Res. 70:373-382.

Bobe, G., J. W. Young, and D. C. Beitz. 2004. Invited review: Pathology, etiology, prevention, and treatment of fatty liver in dairy cows. J. Dairy Sci. 87:3105-3124.

Broadhurst, D. I., and D. B. Kell. 2007. Statistical strategies for avoiding false discoveries in metabolomics and related experiments. Metabolomics 2:171-196.

Danne, O., C. Lueders, C. Storm, U. Frei, and M. Möckel. 2007. Whole blood choline and plasma choline in acute coronary syndromes: Prognostic and pathophysiological implications. Clin. Chim. Acta 383:103-109.

Dervishi, E., G. Zhang, S. M. Dunn, R. Mandal, D. S. Wishart, and B. N. Ametaj. 2017. GC-MS metabolomics identifies metabolite alterations that precede subclinical mastitis in the blood of transition dairy cows. J. Proteome Res. 16:433-446.

Dervishi, E., G. Zhang, D. Hailemariam, S. M. Dunn, and B. N. Ametaj. 2015. Innate immunity and carbohydrate metabolism alterations precede occurrence of subclinical mastitis in transition dairy cows. J. Anim. Sci. Technol. 57:46.

Drackley, J. K. 1999. Biology of dairy cows during the transition period: The final frontier? J. Dairy Sci. 82:2259-2273.
Drobnik, W., G. Liebisch, F.-X. Audebert, D. Frohlich, T. Gluck, P. Vogel, G. Rothe, and G. Schmitz. 2003. Plasma ceramide and lysophosphatidylcholine inversely correlate with mortality in sepsis patients. J. Lipid Res. 44:754-761.

Ducker, G. S., and J. D. Rabinowitz. 2017. One-carbon metabolism in health and disease. Cell Metab. 25:27-42.

Eberhardt, R. 1996. Current Concepts of Bovine Mastitis. 4th ed. National Mastitis Council, Arlington VA.

Edmonson, A., I. Lean, L. Weaver, T. Farver, and G. Webster. 1989. A body condition scoring chart for Holstein dairy cows. J. Dairy Sci. 72:68-78.

Ejigu, B. A., D. Valkenborg, G. Baggerman, M. Vanaerschot, E. Witters, J.-C. Dujardin, T. Burzykowski, and M. Berg. 2013. Evaluation of normalization methods to pave the way towards largescale LC-MS-based metabolomics profiling experiments. OMICS 17:473-485.

Fadden, A. N., and G. Bobe. 2015. Serum visfatin is a predictive indicator of retained placenta and other diseases in dairy cows. J. Vet. Sci. Med. Diagn. 5:1-6.

Franceschi, C., M. Capri, D. Monti, S. Giunta, F. Olivieri, F. Sevini, M. Panagiota Panourgia, L. Invidia, L. Celani, M. Scurti, E. Cavenini, G. C. Castellani, and S. Salvioli. 2007. Inflammaging and anti-inflammaging: A systemic perspective on aging and longevity emerged from studies in humans. Mech. Ageing Dev. 128:92-105.

Geenes, V., and C. Williamson. 2009. Intrahepatic cholestasis of pregnancy. World J. Gastroenterol. 15:2049-2066.

Gopal, P. K., and H. S. Gill. 2000. Oligosaccharides and glycoconjugates in bovine milk and colostrum. Br. J. Nutr. 84:S69-S74.

Gray, R. G., E. Kousta, M. I. McCarthy, I. F. Godsland, S. Venkatesan, V. Anyaoku, and D. G. Johnston. 2013. Ethnic variation in the activity of lipid desaturases and their relationships with cardiovascular risk factors in control women and an at-risk group with previous gestational diabetes mellitus: a cross-sectional study. Lipids Health Dis. 12:25.

Griffith, O. W. 1987. Mammalian sulfur amino acid metabolism: An overview. Methods Enzymol. 143:366-376.

Gruys, E., M. J. M. Toussaint, N. Upragarin, E. A. Van, A. A. Adewuyi, D. Candiani, T. K. Nguyen, and J. Sabeckiene. 2005. Acute phase reactants, challenge in the near future of animal production and veterinary medicine. J. Zhejiang Univ. Sci. B 6:941-947.

Hailemariam, D., R. Mandal, F. Saleem, S. M. Dunn, D. S. Wishart, B. N. Ametaj, Y. Yokomizo, M. Kreutz, L. Fonsmark, P. L. Madsen, and P. Klarlund. 2014. Identification of predictive biomarkers of disease state in transition dairy cows. J. Dairy Sci. 97:2680-2693.

Huber, K., S. Dänicke, J. Rehage, H. Sauerwein, W. Otto, U. RolleKampczyk, and M. von Bergen. 2016. Metabotypes with properly functioning mitochondria and anti-inflammation predict extended productive life span in dairy cows. Sci. Rep. 6:24642.

Hurley, W. L., and P. K. Theil. 2011. Perspectives on immunoglobulins in colostrum and milk. Nutrients 3:442-474.

Imhasly, S., C. Bieli, H. Naegeli, L. Nyström, M. Ruetten, and C. Gerspach. 2015. Blood plasma lipidome profile of dairy cows during the transition period. BMC Vet. Res. 11:252.

Ingvartsen, K. L. 2006. Feeding- and management-related diseases in the transition cow: physiological adaptations around calving and strategies to reduce feeding-related diseases. Anim. Feed Sci. Technol. 126:175-213.

Kelton, D. F., K. D. Lissemore, and R. E. Martin. 1998. Recommendations for recording and calculating the incidence of selected clinical diseases of dairy cattle. J. Dairy Sci. 81:2502-2509.

Kessler, E. C., J. J. Gross, R. M. Bruckmaier, and C. Albrecht. 2014 Cholesterol metabolism, transport, and hepatic regulation in dairy cows during transition and early lactation. J. Dairy Sci. 97:54815490 .

Kirkwood, J. S., C. Maier, and J. F. Stevens. 2013. Simultaneous, untargeted metabolic profiling of polar and nonpolar metabolites by LC-Q-TOF mass spectrometry. Curr. Protoc. Toxicol. 56:4.39.14.39.12. https://doi.org/10.1002/0471140856.tx0439s56.

Kontush, A., M. Lhomme, and M. J. Chapman. 2013. Unraveling the complexities of the HDL lipidome. J. Lipid Res. 54:2950-2963. 
Koves, T. R., J. R. Ussher, R. C. Noland, D. Slentz, M. Mosedale, O. Ilkayeva, J. Bain, R. Stevens, J. R. B. Dyck, C. B. Newgard, G. D. Lopaschuk, and D. M. Muoio. 2008. Mitochondrial overload and incomplete fatty acid oxidation contribute to skeletal muscle insulin resistance. Cell Metab. 7:45-56.

Kuhla, B., G. Nürnberg, D. Albrecht, S. Görs, H. M. Hammon, and C. C. Metges. 2011. Involvement of skeletal muscle protein, glycogen, and fat metabolism in the adaptation on early lactation of dairy cows. J. Proteome Res. 10:4252-4262.

Lai, I. H., J. H. Tsao, Y. P. Lu, J. W. Lee, X. Zhao, F. L. Chien, and S. J. Mao. 2009. Neutrophils as one of the major haptoglobin sources in mastitis affected milk. Vet. Res. 40:17.

Lammert, F., H. U. Marschall, A. Glantz, and S. Matern. 2000. Intrahepatic cholestasis of pregnancy: molecular pathogenesis, diagnosis and management. J. Hepatol. 33:1012-1021.

Lee, Y. S., Y. Cho, and M.-J. Shin. 2015. Dietary very long chain saturated fatty acids and metabolic factors: findings from the Korea National Health and Nutrition Examination Survey 2013. Clin. Nutr. Res. 4:182-189

Lin, Z., Z. Zhang, H. Lu, Y. Jin, L. Yu, and Y. Liang. 2014. Joint MSbased platforms for comprehensive comparison of rat plasma and serum metabolic profiling. Biomed. Chromatogr. 28:1235-1245.

López-Otín, C., M. A. Blasco, L. Partiridge, M. Serrano, and G. Kroemer. 2013. The hallmarks of aging. Cell 153:1194-1217.

Mackenzie, D. D. S., and A. K. Lascelles. 1968. The transfer of $\left.{ }^{131} \mathrm{I}\right]$ labelled immunoglobulins and serum albumin from blood into milk of lactating ewes. Aust. J. Exp. Biol. Med. Sci. 46:285-294.

Malik, V. S., S. E. Chiuve, H. Campos, E. B. Rimm, D. Mozaffarian, F. B. Hu, and Q. Sun. 2015. Circulating very-long chain saturated fatty acids and incident coronary heart disease in U.S. men and women. Circulation 132:260-268.

Mansor, R., W. Mullen, A. Albalat, P. Zerefos, H. Mischak, D. C. Barrett, A. Biggs, and P. D. Eckersall. 2013. A peptidomic approach to biomarker discovery for bovine mastitis. J. Proteomics 85:89-98

Moyes, K. M., T. Larsen, and K. L. Ingvartsen. 2013. Generation of an index for physiological imbalance and its use as a predictor of primary disease in dairy cows during early lactation. J. Dairy Sci. 96:2161-2170.

Nakamura, T., H. Kawase, K. Kimura, Y. Watanabe, M. Ohtani, I. Arai, and T. Urashima. 2003. Concentrations of sialyloligosaccharides in bovine colostrum and milk during the prepartum and early lactation. J. Dairy Sci. 86:1315-1320.

NRC. 2001. Nutrient Requirements of Dairy Cattle. 7th rev. ed. Natl. Acad. Sci., Washington, DC.

R Core Team. 2013. R: A language and environment for statistical computing. R Foundation for Statistical Computing, Vienna, Austria. http://www.R-project.org/.

Qu, Y., A. N. Fadden, M. G. Traber, and G. Bobe. 2014. Potential risk indicators of retained placenta and other diseases in multiparous cows. J. Dairy Sci. 97:4151-4165.

Qu, Y., K. Lytle, M. G. Traber, and G. Bobe. 2013. Depleted serum vitamin E concentrations precede left displaced abomasum in early-lactation dairy cows. J. Dairy Sci. 96:3012-3022.

Rollin, E., K. C. Dhuyvetter, and M. W. Overton. 2015. The cost of clinical mastitis in the first 30 days of lactation: an economic modeling tool. Prev. Vet. Med. 122:257-264.

Rukkwamsuk, T., M. J. H. Geelen, T. A. M. Kruip, and T. Wensing. 2000. Interrelation of fatty acid composition in adipose tissue, serum, and liver of dairy cows during the development of fatty liver postpartum. J. Dairy Sci. 83:52-59.

Servillo, L., A. Giovane, D. Cautela, D. Castaldo, and M. L. Balestrieri. 2014. Where does $\mathrm{N}(\varepsilon)$-trimethyllysine for the carnitine biosynthesis in mammals come from? PLoS One 9:e84589.

Sharma, N., N. K. Singh, and M. S. Bhadwal. 2011. Relationship of somatic cell count and mastitis: an overview. Asian-australas. J. Anim. Sci. 24:429-438.

Silbernagel, G., M. Kovarova, A. Cega, J. Machann, F. Schick, R. Legmann, H. U. Häring, N. Stefan, E. Schlecher, A. Fritsche, and A. Peter. 2012. High heptaic SCD1 activity is associated with low liver fat content in healthy subjects under a lipogenic diet. J. Clin. Endocrinol. Metab. 97:E2288-E2292.

Sordillo, L. M., G. A. Contreras, and S. L. Aitken. 2009. Metabolic factors affecting the inflammatory response of periparturient dairy cows. Anim. Health Res. Rev. 10:53-63.

Stefan, N., A. Peter, A. Cegan, H. Staeger, J. Machann, F. Schick, C. D. Claussen, A. Fritsche, H. U. Häring, and E. Schleicher. 2008. Low heaptic stearoyl-CoA desaturase 1 activity is associated with fatty liver and insulin resistance in obese humans. Diabetologia 51:648-656.

Sundekilde, U. K., N. A. Poulsen, L. B. Larsen, and H. C. Bertram. 2013. Nuclear magnetic resonance metabonomics reveals strong association between milk metabolites and somatic cell count in bovine milk. J. Dairy Sci. 96:290-299.

Tai, C. C., and S. T. Ding. 2010. N-3 polyunsaturated fatty acids regulate lipid metabolism through several inflammation mediators: mechanisms and implications for obesity prevention. J. Nutr. Biochem. 21:357-363.

Tao, N., E. J. DePeters, J. B. German, R. Grimm, and C. B. Lebrilla. 2009. Variations in bovine milk oligosaccharides during early and middle lactation stages analyzed by high-performance liquid chromatography-chip/mass spectrometry. J. Dairy Sci. 92:2991-3001.

ten Bruggencate, S. J., I. M. Bovee-Oudenhoven, A. L. Feitsma, E. van Hoffen, and M. H. Schoterman. 2014. Functional role and mechanisms of sialyllactose and other sialylated milk oligosaccharides. Nutr. Rev. 72:377-389.

Thomas, F. C., M. Mudaliar, R. Tassi, T. N. McNeilly, R. Burchmore, K. Burgess, P. Herzyk, R. N. Zadoks, and P. D. Eckersall. 2016. Mastitomics, the integrated omics of bovine milk in an experimental model of Streptococcus uberis mastitis: 3. Untargeted metabolomics. Mol. Biosyst. 12:2762-2769.

USDA. 2009. Dairy 2007, Part V: Changes in Dairy Cattle Health and Mangagement Practices in the United States, 1996-2007. USDA: APHIS:VS, CEAH. Fort Collins, CO.

Viguier, C., S. Arora, N. Gilmartin, K. Welbeck, R. O'Kennedy, and R. O'Kennedy. 2009. Mastitis detection: Current trends and future perspectives. Trends Biotechnol. 27:486-493.

Wang, Y.-D., W.-D. Chen, M. Wang, D. Yu, B. M. Forman, and W. Huang. 2008. Farnesoid X receptor antagonizes nuclear factor kappa B in hepatic inflammatory response. Hepatology 48:1632-1643.

Xi, X., L.-Y. Kwok, Y. Wang, C. Ma, Z. Mi, and H. Zhang. 2017. Ultra-performance liquid chromatography-quadrupole-time of flight mass spectrometry MS E -based untargeted milk metabolomics in dairy cows with subclinical or clinical mastitis. J. Dairy Sci. 100:4884-4896.

Xia, J., R. Mandal, I. V. Sinelnikov, D. Broadhurst, and D. S. Wishart. 2012. MetaboAnalyst 2.0-a comprehensive server for metabolomic data analysis. Nucleic Acids Res. 40:W127-33.

Yan, J., X. Jiang, A. A. West, C. A. Perry, O. V. Malysheva, S. Devapatla, E. Pressman, F. Vermeylen, S. P. Stabler, R. H. Allen, and M. A. Caudill. 2012. Maternal choline intake modulates maternal and fetal biomarkers of choline metabolism in humans. Am. J. Clin. Nutr. 95:1060-1071.

Yu, Z., G. Kastenmüller, Y. He, P. Belcredi, G. Möller, C. Prehn, J. Mendes, S. Wahl, W. Roemisch-Margl, U. Ceglarek, A. Polonikov, N. Dahmen, H. Prokisch, L. Sie, Y. Li, H.-E. Wichmann, A. Peters, F. Kronenberg, K. Suhre, J. Adamski, T. Illig, and R. Wang-Sattler. 2011. Differences between human plasma and serum metabolite profiles. PLoS One 6:e21230.

Zhang, G., E. Dervishi, S. M. Dunn, R. Mandal, P. Liu, B. Han, D. S. Wishart, and B. N. Ametaj. 2017. Metabotyping reveals distinct metabolic alterations in ketotic cows and identifies early predictive serum biomarkers for the risk of disease. Metabolomics 13:43.

Zhou, Z., T. A. Garrow, Z. Dong, D. N. Luchini, and J. J. Loor. 2017. Hepatic activity and transcription of betaine-homocystein methyltransferase, methionine synthase, and cystathionine synthase in periparturient dairy cows are altered to different extents by methionine and choline. J. Nutr. 147:11-19. 